\title{
Numerical Investigation of Boundary Layer Effects on Vortex shedding Frequency and Forces acted on Marine Pipeline
}

M. H. Kazeminezhad, A. Yeganeh-Bakhtiary, A. Etemad-shahidi

School of Civil Eng., Iran University of Science and Technology, Narmak, Tehran, Iran, P.O. Box 16765-163

\begin{abstract}
In this study the force components and vortex shedding frequency of a pipe exposed to a steady current were numerically investigated in terms of the drag coefficient, lift coefficient and Strouhal number. The effects of the bed proximity and boundary layer thickness on the mentioned parameters were studied extensively. The Reynolds-AveragedNavier-Stokes equations with a $k$ - $\varepsilon$ turbulence closure model were numerically solved to approximate both the flow pattern and pressure distribution around the pipe. The instantaneous drag and lift coefficients were calculated based on the pressure distribution around the pipe, while the Strouhal number was estimated by the spectral analysis of the predicted instantaneous lift force. Evaluation of the numerical model revealed that the model well predicted the velocity profile around the pipe, force coefficients and Strouhal number. The results showed that the model slightly overpredicts the mean force coefficients and Strouhal number. It is concluded that the mean force coefficients and the root-mean-square (RMS) lift coefficient are strongly affected by the gap to diameter ratio while the Strouhal number is slightly affected by the gap ratio. The results also indicated
\end{abstract}


that the mean lift force acted on the pipe is upward in all boundary layers and rapidly decreased by increasing the gap ratio, whereas the mean drag force slightly increased as the gap ratio increased up to a certain value. The RMS lift force also increased as the gap ratio increased up to a certain value and remained approximately constant by further increasing of gap ratio. The mean force coefficients and Strouhal number calculated in terms of the flow velocity at the pipe axis marginally influenced by the boundary layer thickness, while the parameters calculated in terms of the free-stream velocity strongly affected by the boundary layer thickness.

Keywords: Boundary layer thickness, mean drag coefficient, gap ratio, $k-\varepsilon$ turbulence model, mean lift coefficient, RMS lift coefficient, RANS equations, Strouhal Number. 


\section{Introduction}

Submarine pipelines are vital arteries of fluid and energy transportation due to the increasingly need of human to the oil and its byproducts. Offshore pipelines are either laid on or buried in the bed. Pipelines laid on an erodible seabed may face very severe local scouring induced by the flow action, leading to free spanning of the pipeline. Hydrodynamic forces acting on the marine pipelines are one of the important design considerations in the pipeline engineering. The hydrodynamic forces are resulted from the flow action and expressed by two components; one in-line with the flow (drag force) and the other perpendicular to the flow (lift force). These forces have an unsteady nature and oscillate around their average values because of the occurrence of the vortex shedding at the downstream side of the pipe. Vortex shedding leads to the Vortex-Induced-Vibration (VIV) which is an important cause of fatigue damage of pipeline at the free spanning sections [1]. To consider the unsteady nature of the forces and take the VIV effects into account, the flow pattern around the pipe should be simulated accurately. The flow pattern around pipeline and consequently the above mentioned non-dimensional parameters are affected by different parameters such as the bed proximity in terms of gap $(e)$ to pipe diameter $(D)$ ratio, boundary layer thickness $(\delta)$ and pipe's Reynolds number $\left(R e_{p}=u_{0} D / v\right.$, $u_{0}$ is the free-stream velocity and $v$ is the kinematic viscosity) [2].

The drag and lift forces and vortex shedding frequency are usually expressed in nondimensional forms in terms of the drag coefficient $\left(C_{D}\right)$, lift coefficient $\left(C_{L}\right)$ and Strouhal number (St). In the literature, two different definitions of the force coefficients have been used for the pipe. In the former definition, the forces are normalized by the free-stream velocity $\left(u_{0}\right)$ as follows: 


$$
C_{D 0}=\frac{F_{D}}{\frac{1}{2} \rho u_{0}^{2} D} \quad, \quad C_{L 0}=\frac{F_{L}}{\frac{1}{2} \rho u_{0}^{2} D}
$$

while in the second definition, the undisturbed velocity at the pipe axis $\left(u_{a}\right)$ is used to normalize the forces.

$$
C_{D}=\frac{F_{D}}{\frac{1}{2} \rho u_{a}^{2} D} \quad, \quad C_{L}=\frac{F_{L}}{\frac{1}{2} \rho u_{a}^{2} D}
$$

In the above equations $F_{D}, F_{L}$ and $\rho$ are drag force, lift force and fluid density, respectively. When the pipe center locates out of the bed boundary layer, $u_{a}$ becomes equal to $u_{0}$ and these two definitions become equal to each other.

Effects of different parameters on the force coefficients and Strouhal number have been experimentally investigated by many researchers. Kiya [3] and Roshko et al. [4] investigated the drag coefficient with various gap ratios for pipe's Reynolds number of 1$4 \times 10^{4}$ and $2 \times 10^{4}$, respectively. They found that the drag coefficient increases with increasing gap ratio up to a certain value of $(e / D)_{\mathrm{s}}$, and then remains reasonably constant for further increase in $e / D$. Kiya [3] found that $(e / D)_{s}=0.4-0.5$, while Roshko et al. [4] noted that the $(e / D)_{s}$ is apparently equal to the approaching boundary layer thickness. The proposed value for $(e / D)_{\mathrm{s}}$ by Roshko et al. [4] was not confirmed by Buresti and Launaro [5] for laminar boundary layer.

Jones [6] and later Fredsøe et al. [7] investigated the lift coefficient experimentally, and mentioned the lift coefficient strongly depends on the gap ratio and approaching flow. The lift coefficient for a pipeline in a shear-free flow decreases as the gap ratio increases. In shear flow, the lift coefficient is smaller than that of in shear-free flow. 
Zdravkovich [8] measured the force coefficients at Reynolds numbers from $6 \times 10^{4}$ to $1.5 \times 10^{5}$ for different boundary layers that artificially thickened by attaching a tripping rod or rough mesh along the bed. He concluded that the drag coefficient is constant when the pipe located out of the boundary layer and it starts to decrease once the pipe is immersed in the boundary layer. He found different variations of the lift coefficient against $e / D$ for two types of the boundary layers employed in the experiments. In other words, when the pipe was immersed in the boundary layer generated by the rough mesh, negative lift coefficient was observed in the range of $0.2<e / D<0.6$. For the rod generated boundary layer cases, the lift coefficient decreased as the gap ratio increased.

Jensen et al. [9] investigated the variation of the force coefficients and Strouhal number with respect to the gap ratio for Reynolds number of $1 \times 10^{4}$. The obtained results for the drag coefficients were approximately similar to those of Kiya [3]. They found that the lift coefficient strongly depends on the gap ratio and it decreases with the increasing of the gap ratio. Contrary to the lift coefficient, the drag coefficient and Strouhal number were not affected strongly by the gap ratio and they slightly increased as the gap ratio increased. They also found that the fluctuating lift force increases as the gap ratio increases.

Buresti and Lanciotti [10] examined experimentally the force coefficients in natural, rod and mesh generated boundary layers. In their experiments the pipe's Reynolds number varied from $8.6 \times 10^{4}$ to $2.77 \times 10^{5}$. They noticed that the lift coefficient decreases more rapidly in thicker boundary layer as the pipe is moved away from the bed. They also found that the drag coefficient in the thin natural boundary layer increases as the gap ratio increases up to a certain value of $e / D$ and it slightly reduces for further increase in $e / D$. Lei et al. [11] investigated the variation of the force coefficients $\left(C_{D 0}\right.$ and $\left.C_{L 0}\right)$ in different natural and rod generated boundary layer for Reynolds number of about $1.35 \times 10^{4}$. They 
found that the boundary layer thickness marginally affects the drag coefficient, while no monotonic result was observed in different boundary layers. The negative lift was observed for the artificial rod generated boundary layers at gap ratios $e / D=0.4-1$. . They also studied the variation of the RMS lift force with the gap ratio. For the gap ratio between $0-0.2$, the RMS lift force and its variations with $e / D$ were very small. In the range of $0.3<e / D<0.8$, the RMS lift increased strongly with the gap ratio. The RMS lift force remained constant or slightly decreased for further increase in $e / D$.

The Strouhal number and vortex shedding suppression were studied in many studies [e.g. 10, 11, and 12]. There is a good agreement between the previous studies that the critical gap ratio, at which the vortex shedding disappears, decreases with increasing the boundary layer thickness. Generally, the Strouhal number increases as the gap ratio increases up to a certain value and it approximately remains constant $[11,12]$ or slightly decreases $[13,14]$ for further increase of the gap ratio.

In several numerical studies, the force coefficients and Strouhal number for a pipeline were estimated. Numerical estimation of these parameters strongly depends on the modeling of the flow pattern around pipe. Lee et al. [15] and Li et al. [16] solved the Navier-Stokes equations with a SGS (Smagorinsky's subgrid scale) turbulence closure model to simulate the flow behind a pipe. Qualitative comparisons showed favorable agreement with the experimental measurement of Bearman and Zdravkovich [17]. Brørs [18] simulated the flow pattern around the pipe using a standard $k-\varepsilon$ turbulence model. He calculated the drag and lift coefficients for a pipe with $e / D=1$ and $R e_{p}=15000$. The predicted drag coefficient $(0.72)$ was in the middle range of the experimental data whereas the predicted lift coefficient (1.16) was predicted too high compared with the experimental data. Lei et al. [19] successfully employed the Navier-Stokes equations to inquire the 
vortex shedding flow over a circular cylinder near a wall at various gap ratios for different Reynolds numbers from 80 to 1000 . The numerical results showed that as the gap ratio decreases the regularity as well as the amplitude of the instantaneous lift decreases. Dipankar and Sengupta [20] solved the Navier-Stokes equations in vorticity-stream function formulation to estimate the force components and Strouhal number for two different gap ratios of 0.5 and 1.5 with Reynolds number of 1200 . The computed force coefficients and the vortex shedding behavior were qualitatively in good agreement with the previous experimental results. Liang and Cheng [21] simulated the flow pattern around a cylinder exposed to steady current using different turbulence models. They computed the drag and lift coefficients to investigate the dependence of different turbulence models on the computational mesh. They showed the $k-\varepsilon$ and $k-\omega$ turbulence models underpredict the mean drag coefficient. Smith and Foster [22] also investigated the flow hydrodynamic around a pipeline subjected to steady flow for five stages of the scour process. They found that the $k-\varepsilon$ turbulence model is capable of modeling the velocity profile near an offshore pipeline. They also noticed that the $k$ - $\varepsilon$ model is not capable of modeling the strong vortex shedding for a cylinder over the scoured bed. Kazeminezhad et al. [23] investigated the gap ratio effects on the drag force and occurrence of the vortex shedding. They found that the vortex shedding phenomenon and drag coefficient oscillation can be simulated using the $k-\varepsilon$ turbulence model. Guo et al. [24] adopted the $k-\varepsilon$ turbulent model and vorticitystream function formulation to simulate the flow field and the lift and drag coefficients of the circular cylinder. They expressed that the proposed approach is capable of simulating the instantaneous drag and lift forces and flow pattern around a horizontal cylinder. Although the effects of gap ratio on the force coefficients and Strouhal number have been investigated in several experimental studies, some inconsistencies are observed in the 
reported results. For example Lei et al. [11] reported a downward (negative) mean lift force for a pipe immersed in thick boundary layer ( $\delta / D=1.64$ and 2.89) with Reynolds number of $1.3 \times 10^{4}$; whereas, Jensen et al. [9] reported an upward mean lift forces for an approximately identical conditions $\left(\delta / D=2.0, \mathrm{Re}_{\mathrm{p}}=1 \times 10^{4}\right)$. These inconsistencies may partly be due to the difference in the flow characteristics i.e., the boundary layer thickness and Reynolds number. The other part of the observed inconsistencies may arise from the different definitions of the drag and lift coefficients and Strouhal number used in the literature. Hence, the main aim of this study is to find the effects of the gap ratio and boundary layer thickness on the force coefficients and Strouhal number for a marine pipeline exposed to the steady current. This study mainly focused on the simulation of the force coefficients of the pipeline for different gap ratios and boundary layers. To do so, the flow hydrodynamics around a pipeline subjected to the steady flow is simulated by solving Reynolds-Averaged-Navier-Stokes (RANS) equations using a $k-\varepsilon$ model as the turbulence closure model. Various gap ratios and boundary layer thicknesses are systematically considered in the simulations. The model is firstly evaluated against simulation of vortex shedding and velocity profiles around the pipe. The mean drag and lift and RMS lift coefficients are then calculated based on the pressure distribution. The Strouhal number is finally calculated by analyzing the power spectral of the instantaneous lift force.

\section{Governing Equations}

In this study the flow field near a marine pipeline is modeled using the incompressible two dimensional Reynolds-Averaged Navier-Stokes (RANS) equations:

$$
\frac{\partial u_{i}}{\partial x_{i}}=0
$$


$\frac{\partial u_{i}}{\partial t}+u_{j} \frac{\partial u_{i}}{\partial x_{j}}=-\frac{1}{\rho} \frac{\partial p}{\partial x_{i}}+v \frac{\partial}{\partial x_{j}}\left[\frac{\partial u_{i}}{\partial x_{j}}+\frac{\partial u_{j}}{\partial x_{i}}\right]-\frac{\partial \overline{u_{i}^{\prime} u_{j}^{\prime}}}{\partial x_{j}}$

where $x_{i}(i=1,2)$ denotes the Cartesian coordinate system, $x_{1}$ and $x_{2}$ axis are in the horizontal $(x)$ and vertical direction ( $z$ ), respectively. In the above equations $t$ denotes time marching, $u_{j}$ and $p$ denote the $x_{j}$ components of velocities and full pressure, $v$ denotes kinematics viscosity and $\overline{u_{i}^{\prime} u_{j}^{\prime}}$ denotes Reynolds stress tensor. The Reynolds stress is modeled by Boussinesq approximation as follows:

$-\overline{u_{i}^{\prime} u_{j}^{\prime}}=v_{t}\left(\frac{\partial u_{i}}{\partial x_{j}}+\frac{\partial u_{j}}{\partial x_{i}}\right)-\frac{2}{3} k \delta_{i j}$

in which $v_{t}$ is the eddy viscosity, $k$ is the turbulent kinetic energy and $\delta_{i j}$ is the Kronecker delta. Liang and Cheng [21] and Smith and Foster [22] showed that the $k$ - $\varepsilon$ turbulence model is capable of simulating the flow pattern around pipeline. Therefore, the $k-\varepsilon$ model is utilized for the transport closure scheme.

$$
\begin{aligned}
& \frac{\partial k}{\partial t}+u_{j} \frac{\partial k}{\partial x_{j}}=\frac{\partial}{\partial x_{j}}\left[\left(v+\frac{v_{t}}{\sigma_{k}}\right) \frac{\partial k}{\partial x_{j}}\right]+P_{k}-\varepsilon \\
& \frac{\partial \varepsilon}{\partial t}+u_{j} \frac{\partial \varepsilon}{\partial x_{j}}=\frac{\partial}{\partial x_{j}}\left[\left(v+\frac{v_{t}}{\sigma_{\varepsilon}}\right) \frac{\partial \varepsilon}{\partial x_{j}}\right]+\left[c_{\varepsilon 1} P_{k}-c_{\varepsilon 2} \varepsilon\right] \frac{\varepsilon}{k} \\
& P_{k}=v_{t} \frac{\partial u_{i}}{\partial x_{j}}\left(\frac{\partial u_{i}}{\partial x_{j}}+\frac{\partial u_{j}}{\partial x_{i}}\right)
\end{aligned}
$$

where $\varepsilon$ and $P_{k}$ represent dissipation rate of turbulent kinetic energy and production of $k$, respectively. The eddy viscosity is determined using the $k$ and $\varepsilon$ values:

$$
v_{t}=c_{\mu} \frac{k^{2}}{\varepsilon}
$$

The standard $k-\varepsilon$ model coefficients are set according to Launder and Spalding [25]: 
$C_{\varepsilon 1}=1.44, C_{\varepsilon 2}=1.92, \sigma_{\varepsilon}=1.30, \sigma_{k}=1.00, c_{\mu}=0.09$

\section{Boundary Conditions and Numerical Method}

In this study, the laboratory conditions of Jensen [26] and Oner et al. [12] are modeled using a $30 D$ long and $7.5 D$ high rectangular domain. In these cases, the gap ratio was small and the vortex shedding was not as strong as that behind an isolated cylinder. Therefore, it is expected that the computational domain with height of $7.5 \mathrm{D}$ is appropriate for simulation of these cases. In the cases with large gap ratio ( $e / D>0.5)$, the water surface was assumed $10 D$ far from the pipe center.

Fig. 1 displays schematically the computational domain and pipe position. As can be seen, the pipe center is located at $e^{+} D / 2$ distance above the plane bed where $e$ is the gap between pipe surface and plane bed. The inlet and outlet boundaries are located at $10 \mathrm{D}$ and $20 D$ far from the pipe center, respectively. At the inlet boundary, a fully developed turbulent velocity profile is applied:

$u(z)= \begin{cases}\frac{u_{*}^{2} y}{v} & \frac{u_{*} y}{v}<11.63 \\ \frac{u_{*}}{\kappa} \ln \left(E \frac{u_{*} y}{v}\right) & \frac{u_{*} y}{v} \geq 11.63\end{cases}$

$v(z)=0$

$k(z)=\max \left\{C_{\mu}^{-1 / 2}\left(1-\frac{z}{\delta}\right)^{2} u_{*}^{2}, 0.0005 u_{0}^{2}\right\}$

$\varepsilon(z)=\frac{C_{\mu}^{3 / 4}(k(z))^{3 / 2}}{\ell}$

$\ell(z)=\min \left\{\kappa y\left(1+1.5 \frac{z}{\delta}\right)^{-1}, C_{\mu} \delta\right\}$ 
in which $u_{*}$ is the shear velocity, $E$ is a roughness parameter that is equal to 9.793 for hydraulically smooth wall, $\kappa$ is von Karman constant $(=0.42), u_{0}$ is the free-stream velocity and $\ell(z)$ is the turbulence length scale.

The wall function is employed at the plane boundary and cylinder surface [21, 27] by defining $u_{w}=u$ and $z_{w}=z . \quad u_{w}$ and $z_{w}$ are velocity in the tangential direction of the surface and distance between the surface and the first grid of the mesh from the solid surface. Assuming the local equilibrium region close to the walls results in the following boundary conditions for $k$ and $\varepsilon$ :

$$
k_{w}=\frac{u_{*}^{2}}{\sqrt{C_{\mu}}} \quad \varepsilon_{w}=\frac{u_{*}^{3}}{\kappa Z_{w}}
$$

In this study the pipe surface is assumed as a hydraulically smooth one.

As mentioned before, the water surface is assumed far from the cylinder. Therefore, it can be assumed that the effects of blocking and vortex shedding on the water surface are negligible. According to this assumption, a symmetric boundary condition is used $[18,19$, $21,22]$ by setting the vertical velocity to zero and applying zero normal gradient condition for all other quantities according to Nezu [28]. Zero normal gradients of all parameters except pressure are applied at the outlet boundary.

A staggered mesh is used for discretizing of the governing equations. The scalar variables including pressure, turbulent kinetic energy and dissipation rate are defined at the ordinary nodal point located at the center of the scalar cell. The velocities are defined at the scalar cell faces (at the center of the staggered cell) in between of the ordinary nodal points. The governing equations are solved in a Cartesian coordinate by the finite volume method. The equations are discretized using the fully implicit first-order time marching scheme. The hybrid scheme is applied to discretize the equations, in which the convection terms are 
discretized by the first order upwind scheme; while, the diffusion terms are discretized with the second order central differencing scheme. An Iterative solution methodology based on the SIMPLE algorithm [29] is employed to solve the discretized equations.

The flow field close to the walls and downstream of the pipe is complicated due to the formation and interaction of the shear layers. Therefore, finer meshes are employed in these areas. Fig. 2 shows different parts of the computational domain and maximum dimensionless mesh size $\left(\Delta x^{*}=\Delta x / D, \Delta z^{*}=\Delta z / D\right)$ for the case with a gap ratio equal to zero. It should be noted that 50 grids were used per cylinder diameter in horizontal and vertical directions. The mesh size is gradually increased to reach its maximum values near the downstream, upstream and free surface boundaries. The mentioned mesh resolution was selected by sensitivity analyses which will be discussed in the next section.

\section{Numerical Results and Discussion}

\subsection{Effects of mesh and time step resolutions}

The appropriate mesh size and time step length should be determined prior to simulations. Therefore, different tests were made to check the sensitivity of the model on the mesh and time step resolutions and also to investigate the model convergence. To do so, the flow pattern around a cylinder was simulated for a case with $R e_{p}=9500, e / D=0.5$ and $\delta / D=1.4$

using dimensionless time step $\left(\Delta t^{*}=\frac{u_{0} \Delta t}{D}\right)$ of 0.02 on three mesh systems; coarse mesh (mesh 1), fine mesh (mesh 2) and very fine mesh (mesh 3). Table 1 shows the number of mesh per cylinder diameter and maximum dimensionless mesh size in subdomain 1 (please see Fig. 2). To find the mesh resolution effects on the numerical results, the dimensionless horizontal velocity $\left(u / u_{0}\right)$ at the upper edge of the cylinder wake at $x / D=1$ 
and $y / D=0.5$ (point 1 on Fig. 1) was illustrated in Fig. 3. As can be seen, the predicted velocity amplitude on mesh 1 is less than that on the finer mesh (mesh 2 and mesh 3). Fig. 3 also shows that the simulated velocity on mesh 2 is very close to the simulated one on mesh 3. To further investigate of the mesh resolutions on the simulations, the instantaneous lift force was calculated using different mesh resolutions. According to Fig. 4, the lift force was calculated as follows:

$$
F_{L}=\int_{0}^{2 \pi} p \sin (\phi) r_{0} d \phi
$$

Results indicated that the variation of the lift force simulated on mesh 1 is smaller than that on mesh 2, while there are no significant differences between the obtained results on mesh 2 and mesh 3. Therefore, it can be concluded that mesh 2 is fine enough to ensure that results are not appreciably mesh dependent.

To find the dependency of the results on the time step length, simulation on mesh 2 were repeated with different dimensionless time steps of $0.005,0.02,0.04$, and 0.2 . Fig. 5 shows the simulated $u / u_{0}$ at point 1 for different time step lengths. It is seen that the amplitude of the velocity in the wake flow becomes smaller as the time step becomes greater. Results show no noticeable difference between the obtained results based on $\Delta t^{*}$ of 0.02 and 0.005. To know the sensitivity of the lift force with respect to the selected time step lengths, the instantaneous lift force was also calculated and illustrated in Fig. 6. It is evident that the numerical model can simulate the oscillatory nature of the lift force using small time marching and the lift oscillation decreases as the time marching becomes greater. The figure also indicates that, contrary to the lift oscillation, the mean lift does not depend on the time step. According to the obtained results, the dimensionless time step is set to 0.02 in all simulations. 


\subsection{Model validation}

For accurate estimation of the force components on the pipe, the numerical model should accurately simulate the velocity profile and pressure distribution around the pipe. The model was validated by comparing the simulated and measured velocity profile upstream and downstream of a pipe exposed to the steady current. Laboratory conditions of Oner et al. [12] were firstly considered to validate the model. Oner et al. [12] measured the horizontal velocity profiles around a pipe exposed to the steady current. The tests were undertaken with the different gap ratios and pipe’s Reynolds numbers. The model was evaluated for test cases with $e / D=0$ and 0.2 and $R e_{p}=9500$. In the selected test cases, the free-stream velocity, pipe diameter and boundary layer thickness were $19.7 \mathrm{~cm} / \mathrm{s}, 5 \mathrm{~cm}$ and $7 \mathrm{~cm}$, respectively.

Fig. 7 shows the comparisons of the simulated and measured mean horizontal velocity around the pipe with $e / D=0$ at the various cross-sections. The model describes the horizontal velocity profile at the upstream and downstream of the pipe very well, while it overestimates the mean horizontal velocity at the upper part of the generated wake. Fig. 8 depicts the simulated and measured velocity profile around the pipe placed near the bed with $e / D=0.2$. As can be seen, the numerical model is capable of simulating the mean horizontal velocity profiles around the pipe; however, it slightly overpredicts the fluid velocity under the pipe. The model well describes the existence of the wake downstream of the pipe, while it slightly overestimates the mean horizontal velocity at the upper part of the generated wake. The overestimation of the mean horizontal velocity at the upper shear layer of the cylinder wake flow was also reported by Liang et al. [21] and Smith and Foster [22], where they used the $k-\varepsilon$ turbulence closure. The model's ability in the prediction of the velocity profile was quantified with the root-mean-square-error (RMSE) 
in Table 2. The largest RMSE is seen downstream of the cylinder, where the model overestimates the horizontal velocity profile at the upper shear layer.

The numerical model was secondly compared against the experiments carried out by Jensen [26] to validate the capability of the model in the simulation of mean vertical velocity. In the mentioned experimental work the gap ratio and pipe's Reynolds number were 0.37 and 7000, respectively. Fig. 9 shows the mean velocity profile downstream of the pipe at $1 D$ and $1.5 D$ intervals from the pipe center. In the figure, the velocities and dimensions were normalized by the free-stream velocity and pipe diameter, respectively. Comparison between the simulated and measured velocity profile demonstrates that the model is capable of simulating the mean horizontal and vertical velocity profiles downstream of a pipe where the vortex shedding occurs. Generally, Figs. 7-9 indicate the favorable agreements between the predicted and measured mean velocity around the pipe. One of the most important phenomena that occurs behind the pipe is the vortex shedding. It strongly affects the instantaneous drag and lift forces and results in periodic variations of the force components on the cylinder. It occurs due to the interaction of generated shear layers on both sides of the pipe. If the pipe is placed in close vicinity of a wall, the bottom shear layer is not developed as strongly as the upper shear layer, which leads to a weak interaction between the shear layers and subsequently suppression of the vortex shedding [2]. By considering one of the Oner et al.'s [12] experimental condition in which the gap ratio and pipe’s Reynolds number are 0.3 and 9500, model capability in the simulation of the vortex shedding is examined. It was understood that the model is able to preciously simulate the vortex shedding provided that the dimensionless time step becomes less than 0.04. Applying different mesh resolutions also indicated that the model is not capable of modeling strong vortex shedding on coarse mesh. These results are inline with the 
obtained results in Figs. 3, 5 and 6 where the variation of the horizontal velocity at cylinder wake flow and lift force became smaller as the time and mesh resolutions became greater.

Fig. 10 shows the simulated instantaneous streamline patterns downstream of the pipe at different time instants with 0.2 (s) intervals. The vortex shedding and vortex formation downstream of the pipe is clearly observed in this figure. In Fig. 10- $a$, two vortices are observed, a weak one at the free-stream side and a strong one at the wall-side. As can be seen the free-stream side vortex gradually becomes stronger while the wall-side vortex gradually sheds from the pipe surface. In Fig. 10- $d$ the wall-side vortex is shed and convected downstream by the current flow. Following the shedding of the wall-side vortex, the free-stream vortex becomes stronger and a new vortex is generated at the wallside (Fig. 10-e). After a while, the free-stream vortex is shed (Fig. 10-f). Qualitative comparison of the observed and simulated vortex shedding indicates that the model is capable of simulating the vortex shedding.

\subsection{Investigation of mean force components acting on the pipe}

Generally, the drag force includes the in-line pressure and friction forces (see Fig. 4). Based on Achenbach [30] experimental works, it can be confirmed that the contribution of friction force to the total drag force is less than 2-3\%. Thus, according to Fig. 4 the drag force is calculated as follows:

$$
F_{D}=\int_{0}^{2 \pi} p \cos (\phi) r_{0} d \phi
$$

To see how well the numerical model performs, the mean drag and lift coefficients are calculated for Jensen et al. [9] experimental conditions. They measured the mean drag and 
lift coefficients acting on the pipe with the different gap ratios for $R e_{p}=1 \times 10^{4}$. The boundary layer thickness to pipe diameter ratio was 2 in the experiments. Fig. 11 illustrates the predicted and measured mean drag and lift coefficients for gap ratios of 0 , 0.1, 0.4, 0.5 and 0.7. Numerical and experimental results presented in Fig. 11-a show that the drag coefficient increases gradually as the gap ratio rises up and the increase rate reduces as $e / D$ increases. Table 3 shows the error statistics of the model in the simulation of different parameters. It is seen that the model slightly overpredicts the drag coefficient with a RMSE of the 0.11 .

It should be noted that in this study the pipe surface have been assumed as the hydraulically smooth wall. Therefore, the contribution of friction force to the total drag force is negligible. To investigate the magnitude of the friction force with respect to the inline pressure force, both components are calculated for the case with $e / D=0.5$. The friction force is calculated as follows:

$F_{f}=\int_{0}^{2 \pi} \tau_{0} \sin (\phi) r_{0} d \phi$

in which $\tau_{0}$ is wall shear stress on the cylinder surface. Results indicate that the friction force is about $1.4 \%$ of the in-line pressure force. Therefore, the omission of the friction force in the simulations does not appreciably change the results.

Fig. 11- $b$ compares the predicted and measured lift coefficients for the different gap ratios. As can be seen the model slightly overpredicts the lift coefficient with a RMSE of 0.13 (table 3). Calculated instantaneous drag and lift coefficients reveal that the oscillation of the force components ceases for the cases of $e / D=0,0.1$ due to the suppression of the vortex shedding. 
To investigate the dependence of the hydrodynamic force coefficients on the boundary layer thickness and gap ratio, drag and lift coefficients are predicted for several cases using the validated numerical model. In all cases the pipe diameter, free-stream velocity and pipe’s Reynolds number are kept at $0.05(\mathrm{~m}), 0.197(\mathrm{~m} / \mathrm{s})$ and 9500, respectively. Different gap ratios of $0,0.1,0.2,0.3,0.5,0.7,1.0,1.5$ and 2.0 with various boundary layer thickness ratios $(\delta / D)$ of $0.3,0.5,0.9,1.4,2.0$ and 3.0 are considered. Since in many studies, e.g. $[11,21,27]$, the $C_{D 0}$ and $C_{L 0}$ parameters have been used for expressing the force components acting on the pipe, these parameters are used here. Fig. 12 illustrates the variation of the mean drag coefficient $\left(C_{D 0}\right)$ calculated in terms of the free-stream velocity versus the gap ratios and boundary layers. As can be seen the $C_{D 0}$ gradually increases with the increasing of the gap ratio until $e / D=0.5-0.6$ and then the increase rate reduces by increasing the gap ratio. This is due to the fact that the drag coefficient depends strongly on the base pressure (pressure behind the pipe), which drops quickly and becomes more negative as the pipe moves away from the bed. The mean drag coefficient is reasonably constants for the range of $e / D>0.4-0.5$ in some boundary layers, which is in very good agreement with the experimental results of Kiya [3]. Fig. 12 also shows that for the thin boundary layer, the $C_{D 0}$ reaches to its maximum value at $e / D=0.5$, and it marginally reduced by increasing the gap ratio. Lei et al. [11] and Buresti and Lanciotti [10] also reported that in the natural thin boundary layer, the $C_{D 0}$ reaches to its maximum value at $e / D=0.6-0.7$ and it slightly decreases for further increase of the gap ratio. This is due to the complicate simultaneous effects of gap ratio and boundary layer thickness on the base pressure behind the pipe. Fig. 12 shows that the $C_{D 0}$ slightly increase as the thickness of the boundary layers decreases. Lei et al. [11] also found that in the some ranges of the gap ratios, the $C_{D 0}$ in the thinner boundary layers are slightly greater than those in the thicker 
boundary layer. Buresti and Lanciotti [10] observations confirms the obtained results in the present study, either.

Fig. 13 illustrates the variation of calculated mean lift coefficients $\left(C_{L 0}\right)$ against different gap ratios. It is clearly seen that the $C_{L 0}$ remarkably decreases as the gap ratio increases due to large upward displacement of the front stagnation point. It is evident that for $e / D=0$, the $C_{L 0}$ varies between about 0.6 and 0.83 , having its minimum in the thicker boundary layer. As the pipe is moved away from the bed, the boundary layer effect on the $C_{L 0}$ is decreased. Results also indicate that the $C_{L O}$ increases as the boundary layer thickness decreases, especially for the smaller gap ratios. This is mainly due to the fact that for a specified gap ratio, the front stagnation point moves toward the bed as the boundary layer thickness ratio decreases. Lei et al. [11] and Zdravkovich [8] reported that there is negative downward mean lift force acting on the pipe in artificial thick boundary layers, while in this study the calculated mean lift forces are always upward and positive. On the other hand, Jensen et al. [9] also reported the positive mean lift in thin natural boundary layer for the all range of gap ratio. Therefore, it can be concluded that the obtained negative mean lift forces are due to the artificial generation of the boundary layer and resulted from the presence of the rod or mesh in the approaching flow. Through the comparison of Figs. 12 and 13, it is readily confirmed that the boundary layer more affects the $C_{D 0}$ than $C_{L 0}$ while the gap ratio affects the $C_{L 0}$ than $C_{D 0}$ more.

It is seen that the boundary layer effects on the $C_{D 0}$ and $C_{L 0}$ are relatively high. This is due to the fact that for a specified gap ratio, the flow velocity at the axis of the pipe is not constant in different boundary layers. In other words, when the pipe center is located at the boundary layer, the velocity of the approaching flow which influences the pipe varies with the boundary layer thickness. To contain the effects of boundary layer on the force 
components, it is better to apply the force coefficients calculated in terms of the flow velocity at the pipe axis.

Fig. 14 illustrates the mean drag coefficient $\left(C_{D}\right)$ calculated in terms of the velocity at the pipe center for both different gap ratios and boundary layer thickness. As expected, the mean drag coefficient slowly increases with increase of the gap ratio until $e / D=0.5-0.7$. In the range of $e / D \geq 0.7$, it is seen that the mean drag coefficient decreases very slightly as the $e / D$ increases. For the case of $\delta / D=0.5$, Zdravkovich [8] also indicated that in the range of $e / D>0.6$, the $C_{D}$ slightly decreases with increase of the gap ratio. It also can be seen that in this range, the $C_{D}$ decreases as the boundary layer thickness ratio increases. The comparison of Figs. 12 and 14 indicates that the boundary layer thickness influences the mean drag coefficient more in terms of free-stream velocity than the drag coefficient in terms of the velocity at the pipe center.

Fig. 15 illustrates the mean lift coefficient calculated in terms of the velocity at the pipe center for the various gap ratios and boundary layers. While the average lift coefficient for the gap ratio of 0.3 is equal to 0.1 , it increases enormously as the gap ratio decreases. This is in line with the experimental results of Fredsøe et al. [7]. The largest lift coefficient is predicted for $e / D=0$ which is on average equal to 0.87 . Fig. 15 indicates that the effect of various boundary layers on the mean lift coefficient is more pronounced for the smaller $e / D$. For the range of $e / D>0.6$, it is seen that the mean lift coefficient increases slightly with the decrease of the boundary layer thickness. Comparison of Figs. 14 and 15 shows that the variation of gap ratio has more effect on the mean lift coefficient than the mean drag coefficient. The results also indicate that the variation of boundary layer thickness has more effect on the mean drag coefficient than the mean lift coefficient. Close observation of Figs. 13 and 15 reveals that the boundary layers affect the $C_{L o}$ more than $C_{L}$. This is due 
to the fact that in the latter case, the boundary layer effect is partly considered in terms of the flow velocity at the pipe center.

\subsection{Investigation of the fluctuating lift force}

In the previous section, the effects of gap ratio and boundary layer thickness on the mean drag and lift forces were studied. In the cases with large gap to diameter ratio, where the mean lift force is negligible, the importance of fluctuating lift is more than the mean lift. Therefore, in this section the effects of gap to diameter ratio and boundary layer thickness on the fluctuating lift are studied in term of root-mean-square (RMS) of the fluctuating lift force $\left(C_{L}^{\prime}\right)$. Fig. 11-c compares the simulated and measured [9] RMS lift coefficient for $R e_{p}=10^{4}$ with different gap ratios. It is seen that the model underestimates the $C_{L}^{\prime}$ in the case with $e / D=0.4$, while it overpredicts the $C_{L}^{\prime}$ for cases with larger gap ratios. Underestimation of $C_{L}^{\prime}$ for small gap ratio shows that the model underestimates the vortex shedding intensity in the cases with small gap ratio. This is in agreement with the previous studies [e.g. 21, 22], in which the intensity of vortex shedding underestimated by $k-\varepsilon$ turbulence model for small gap ratios. The model accuracy in the prediction of $C_{L}^{\prime}$ is presented in table 3. As can be seen the average measured, average simulated and RMSE of $C_{L}^{\prime}$ are $0.16,0.17$ and 0.17 , respectively.

Variations of the RMS lift coefficient (calculated in terms of the velocity at the cylinder center) with gap ratio and boundary layer thickness have been shown in Fig. 16 for the previous numerical simulations $\left(R e_{p}=9500\right)$. It is seen that the lift fluctuation ceases at very small gap ratios. The RMS lift coefficient increases with the increasing of the gap ratio up to $e / D=0.9-1.0$. The $\mathrm{RMS}$ lift remains approximately constant or slightly 
decreases for more increase in $e / D$. This is inline with the obtained results by Lei et al. [11]. In the cases with $e / D \leq 0.5$, the $C_{L}^{\prime}$ in the thicker boundary layer is greater than that in the thinner boundary layer. This is due to the fact that the generated vortex shedding in thicker boundary layer is stronger than that in the thinner boundary layer at small gap ratios [11]. Comparisons of Figs. 16 and 15 shows that the boundary layers more affect the RMS lift coefficient than the mean lift coefficient.

\subsection{Investigation of vortex shedding frequency}

Vortex shedding frequency can be determined using the power spectral analysis of the lift force acting on the pipe or the instantaneous flow velocity at a point in the near-wake region of the pipe. The frequencies calculated based on the power spectral analysis of the wake velocities depends on the velocity recording point and may vary as the location of the recording point varies. Therefore, it may not be as accurate as the calculated frequency using the spectral analysis of the lift forces [12]. The vortex shedding frequency usually presents in non-dimensional form in terms of Strouhal number. Similar to the force coefficients, two definitions have been used in the literature for the Strouhal number as follows:

$$
S t_{0}=\frac{f D}{u_{0}} \quad, \quad S t=\frac{f D}{u_{a}}
$$

in which $f$ represents the vortex shedding frequency.

In this section the Strouhal number is firstly determined for Oner et al.'s [12] laboratory conditions. Oner et al. [12] calculated the $S t_{0}$ using power spectral analyses of wake velocity for a flow with $R e_{p}=9500, \delta / D=1.4$ and different gap ratios. In this study, the spectral analysis of the calculated lift force is used to identify the vortex shedding 
suppression point and its frequency. Fig. 17 illustrates the spectral analyses of the calculated lift forces for the different gap ratios of $0.1,0.2,0.3,0.5$ and 1.0. The dominant frequency of the spectrums adequately represents the vortex shedding frequencies. It is seen that there is no clear peak in Fig. 17-a, which means that the vortex shedding does not occur at $e / D=0.1$. In Fig. $17-b$ a weak peak with the frequency of vortex shedding appears which shows the occurrence of a weak irregular vortex shedding at $e / D=0.2$. By increasing the gap ratio, a single sharp peak is observed in the spectrums. Fig. 17-c shows that at $e / D=0.3$, regular vortex shedding with a small intensity occurs. For the range of $e / D \geq 0.5$, a sharp peak with a relatively high intensity can be seen in the spectrums, which mean that the vortex shedding is strong. A comparison between measured and simulated $S t_{0}$ for different gap ratios has been illustrated in Fig. 18. As can be seen there is a very good agreement between the predicted and measured $S t_{0}$. Table 3 shows the error statistics of the model in the prediction of the Strouhal number. It is seen that the model overestimates the Strouhal number with RMSE of 0.014. The model's accuracy in the prediction of $S t_{0}$ decreases by increasing the gap ratio, and the model's accuracy lies in the acceptable range.

To explore the Strouhal number dependency on the gap ratio and boundary layer thickness, the Strouhal number is calculated for the previous numerical simulations. The results indicated that the peak of the spectrums decreases as the boundary layer thickness decreases. Fig. 19 illustrates the variation of the $S t_{0}$ versus gap ratio for different boundary layers. As can be seen, the $S t_{0}$ increases with the increasing of the gap ratio up to a certain value of $e / D$. It is clearly seen that the boundary layer thickness affects the $S t_{0}$ parameters especially in the range of $e / D \leq 1.0$. Variation of $S t$ against different gap ratios and boundary layers are presented in Fig. 20. In the range of $e / D \leq 0.5$ the St decreases with the 
decreasing of the gap ratio, while for $e / D \geq 0.7$ the $S t$ very slightly increases as the gap ratio decreases. This is in agreement with the experimental results of Grass et al. [13] and Raven et al. [14] where they reported that the Strouhal number (St) increases slightly with decreasing of the gap ratio in their measurements ranged $0.5 \leq e / D \leq 6$. It is seen that the boundary layer thickness dose not strongly affect the St parameter, while it strongly affects the $S t_{0}$ parameter.

\section{Summary and Conclusions}

In this study, the forces acting on the pipe and the vortex shedding frequency were numerically investigated in terms of non-dimensional parameters e.g. drag coefficient, lift coefficient, RMS lift coefficient and Strouhal number. The Reynolds-Averaged NavierStokes equations with a $k$ - $\varepsilon$ turbulence closure model were employed to simulate the flow pattern and pressure distribution around the pipeline and subsequently to estimate the fluctuating force coefficients. Two different definitions of the mentioned non-dimensional parameters were considered. In the former definition, the parameters were calculated in terms of the free-stream velocity, while in the latter one the parameters were calculated in terms of the flow velocity at the pipe axis. Comparison between the model results and measurements of Jensen [26], Jensen et al. [9] and Oner et al. [12] confirms that the model well predicts the non-dimensional parameters as well as the mean velocity profile around the pipeline. Effects of the gap ratio and boundary layer thickness on the parameters were examined by considering different gap ratios and boundary layer thickness in the simulations. The following conclusions can be drawn from the present study: 
- The $k-\varepsilon$ turbulence closure model is capable to simulate the vortex shedding phenomena and the fluctuating force components by tuning the mesh and time resolutions in the simulations.

- The developed model slightly overestimates the mean drag coefficient, mean lift coefficient and Strouhal number.

- The mean drag coefficient is slowly increased with increasing the gap ratio up to a certain value of $e / D$. The boundary layer thickness affects more the mean drag coefficients calculated in terms of the free-stream velocity than those calculated in terms of the flow velocity at the pipe axis. The mean drag coefficient calculated in terms of free-stream velocity slightly decreases with the increase of the boundary layer thickness.

- As the pipe moves away from the bed, the mean lift coefficient strongly decreases. The mean lift coefficient calculated in terms of the free-stream velocity is strongly affected by the boundary layer thickness, especially for small range of the gap ratios. The mean lift coefficient calculated in terms of the free-stream velocity slightly decreases as the boundary layer thickness increases.

- In natural boundary layer applied in the present study, the calculated mean lift coefficients are always positive and upward either in thin or thick boundary layers. The observed downward and negative mean lift forces in some experimental works may be due to the artificial generation of the thick boundary layers.

- The RMS lift coefficient increases as the gap ratio increases up to $e / D=0.9-1.0$. For $e / D<0.5$, the RMS lift coefficient decreases with decrease of boundary layer thickness. 
- Spectral analysis of the lift force indicates that the peak of the spectrums increases with increase of the gap ratio or boundary layer thickness. In the all cases, the vortex shedding is not observed when $e / D<0.2$, while a very weak vortex shedding is observed when $e / D=0.2$.

- The boundary layer thickness affected the Strouhal number calculated in terms of the free-stream velocity, while its effect on the Strouhal number calculated in terms of the flow velocity at the pipe center is weak. For $e / D \leq 0.5$ the $S t$ decreases with the decreasing of the gap ratio, while for $e / D \geq 0.7$ the St very slightly increases as the gap ratio decreases.

- The force coefficients and Strouhal number calculated in terms of the flow velocity at the pipe center are less dependent on the boundary layer thickness.

\section{ACKNOWLEDGMENT}

The authors are grateful to three anonymous reviewers for their helpful comments. 


\section{References}

[1] F.P. Gao, B. Yang, Y.X. Wu, S.M. Yan, Steady current induced seabed scour around a vibrating pipeline. Appl. Ocean Res. 28 (2006) 291-298.

[2] B.M. Sumer, J. Fredsøe, Hydrodynamics around cylindrical structures, World Scientific Publishing, Singapore, 1999.

[3] M. Kiya, Study on the turbulent shear flow past a circular cylinder, Bulletin Faculty of Engineering, Hokkaido Univ. 50 (1968) 1-100.

[4] A. Roshko, A. Steinolffron, V. Chattoorgoon, Flow forces on a cylinder near a wall or near another cylinder, Proceeding of 2nd US Conf. wind Engineering Research, Fort Collins (1975), paper IV-15.

[5] G. Buresti, F. Launaro, Pressure measurements around a circular cylinder in cross flow near a plane boundary, Institute of Aeronautics of the Univ. of Pisa (1980) Report AIAA 80.

[6] W.T. Jones, Forces on submarine pipelines from steady currents. Petroleum Mechanical Engineering with Underwater Technology Conference, Houston, Texas, Underwater Technology Div., ASME (1971).

[7] J. Fredsøe, B.M. Sumer, J. Andersen, E.A. Hansen, Transverse vibrations of a cylinder very close to a plane wall. J. Offshore Mech. Arct. Engng. 109 (1985) 52-60.

[8] M.M. Zdravkovich, Forces on a circular cylinder near a plane wall. Appl. Ocean Res. 7 (1985) 197-201.

[9] B.L. Jensen, B.M. Sumer, H.R. Jensen, J. Fredsøe, Flow around and forces on a pipeline near a scoured bed in steady current, J. Offshore Mech. Arct. Engng. 112 (1990) 206-213. 
[10] G. Buresti, A. Lanciotti, Mean and fluctuating forces on a circular cylinder in crossflow near a plane surface, J. of Wind Engng. Ind. Aerodyn. 41 (1992) 639-650.

[11] C. Lei, L. Cheng, K. Kavanagh, Reexamination of the effect of a plane boundary on force and vortex shedding of a circular cylinder, J. of Wind Engng. Ind. Aerodyn. 80-3 (1999) 263- 286.

[12] A.A. Oner, M.S. Kirkgoz, S. Akoz, Interaction of a current with a circular cylinder near a rigid bed. Ocean Engng. 35 (2008) 1492-1504.

[13] A.J. Grass, P.W.J. Raven, R.J. Stuart, J.A. Bray, The Influence of boundary layer velocity gradients and bed proximity on vortex shedding from free spanning pipelines, J. Energy Resources Technology 106 (1984) 70- 78.

[14] P.W.J. Raven, R.J. Stuart, J.A. Bray, P.S. Littlejohns, Full-scale dynamic testing of submarine pipeline spans. 17th Annual Offshore Technology Conference, Houston, Texas, May 6-9 (1985) 395-404.

[15] Y.G. Lee, S.W. Hong, K.J. Kang, A numerical simulation of vortex motion behind a circular cylinder above a horizontal plane boundary, Proceedings of the Fourth International Offshore and Polar Engineering Conference, Osaka, Japan, vol. 3 (1994) 427-433.

[16] Y. Li, B. Chen, G. Lai, The numerical simulation of wave forces on seabed pipeline by three-step finite element method and large eddy simulation, Proceedings of The Seventh International Offshore and Polar Engineering Conference, Honolulu, USA, vol. 2 (1997) 273- 277.

[17] Bearman, P.W. and Zdravkovich, M.M., 1978, Flow around a circular cylinder near a plane boundary, Journal of Fluid Mechanics 89 (1), pp. 33-47.

[18] B. Brørs, Numerical modeling of flow and scour at pipelines. J. Hydraul. 
Engng. 125(5) (1999) 511-523.

[19] C. Lei, L. Cheng, S.W. Armfield, K. Kavanagh, Vortex shedding suppression for flow over a circular cylinder near a plane boundary, Ocean Engng. 27 (2000) 1109-1127. [20] A. Dipankar, T.K. Sengupta, Flow past a circular cylinder in the vicinity of a plane wall, J. Fluids Struct. 20 (2005) 403-423.

[21] D. Liang, L. Cheng, Numerical modeling of flow and scour below a pipeline in currents. Part I: Flow simulation, Coast. Engng. 52 (2005) 25-42.

[22] H.D. Smith, D.L. Foster, Modeling of flow around a cylinder over a scoured bed, J. Waterw. Port Coast. Ocean Engng., ASCE 131(1) (2005) 14-24.

[23] M.H. Kazeminezhad, A. Yeganeh-Bakhtiary, A. Etemad-Shahidi, R. Valipour, Numerical investigation of gap to diameter ratio effects on flow pattern and drag force around offshore pipeline. Proceeding of 27th International Conference On Offshore Mechanics and Arctic Engineering, ASME, Estoril, Portugal (2008).

[24] S.X. Guo, W.P. Li, W. Zhao, B. Chen, Sand accumulation on a wall in flows around a near-wall circular cylinder. Acta Mechanica. 196 (2008) 175-185.

[25] B.E. Launder, D.B. Spalding, The Numerical Computation of Turbulent Flow, J. Comput. Math. Appl. Mech. Engng. 3 (1974) 269-289.

[26] B.L. Jensen, Large-scale vortices in the wake of a cylinder placed near a wall, Proceedings of 2nd international conference on laser anemometry-advances and applications, Strathclyde, UK. (1987) 153- 163.

[27] M. Zhao, L. Cheng, B. Teng, Numerical modeling of flow and hydrodynamic forces around a piggyback pipeline near the seabed. J. Waterw. Port Coast. Ocean Engng, ASCE 133(4). (2007) 286-295. 
[28] I. Nezu, Turbulent structure in open-channel flows, Ph.D. Thesis, Kyoto university (1977).

[29] S.V. Patankar, D.B. Spalding, A calculation procedure for heat, mass and momentum transfer in three-dimensional parabolic flows, J. Heat Mass Transfer. 15 (1972) 17871806.

[30] E. Achenbach, Distribution of local pressure and skin friction around a circular cylinder in cross-flow up to $R e=5 \times 105$. J. Fluid Mech. 34(4) (1968) 625-639. 


\section{Figure captions}

Fig. 1. Sketch of the computational domain.

Fig. 2. Computational domain size and dimensionless mesh size in different sections.

Fig. 3. Simulated instantaneous non-dimensional horizontal velocity at wake flow (point

1) using different mesh resolutions $\left(R e_{p}=9500, e / D=0.5\right)$.

Fig. 4. Total pressure and friction force acting on the pipe [2].

Fig. 5. Simulated instantaneous non-dimensional horizontal velocity at wake flow (point 1) using different time steps in the numerical model $\left(R e_{p}=9500, e / D=0.5\right)$.

Fig. 6. Simulated instantaneous lift coefficient using different time steps in the numerical model $\left(R e_{p}=9500, e / D=0.5\right)$.

Fig. 7. Mean horizontal velocity. The experimental data of [12] are presented by symbols. The solid lines present the model results ( $\left(2 / D=0.0, R e_{p}=9500\right)$.

Fig. 8. Mean horizontal velocity. The experimental data of [12] are presented by symbols. The solid lines present the model results $\left(e / D=0.2, R e_{p}=9500\right)$.

Fig. 9. Mean horizontal and vertical velocity $\left(u / u_{0}\right.$ and $\left.v / u_{0}\right)$. The experimental data of [26] are presented by the square symbols. The solid lines present the model data $(e / D=0.37$, $\left.R e_{p}=7000\right)$.

Fig. 10. Instantaneous streamlines and vortex shedding ( $\left.e / D=0.3, R e_{p}=9500\right)$.

Fig. 11. Comparison of the (a) mean drag, (b) mean lift and (c) RMS lift coefficients between the present model and measurements of Jensen et al. [9] for different gap ratios.

Fig. 12. Drag coefficient in terms of free-stream velocity versus gap ratio for different boundary layers.

Fig. 13. Lift coefficient in terms of free-stream velocity versus gap ratio for different boundary layers. 
Fig. 14. Drag coefficient in terms of flow velocity at the pipe center versus gap ratio for different boundary layers.

Fig. 15. Lift coefficient in terms of flow velocity at the pipe center versus gap ratio for different boundary layers.

Fig. 16. RMS Lift coefficient in terms of flow velocity at the pipe center versus gap ratio for different boundary layers.

Fig. 17. Power spectral density of the lift force for (a) $e / D=0.1,(b) e / D=0.2$, (c) $e / D=0.3$, (d) $e / D=0.5$ and $(e) e / D=1.0\left(\delta / D=1.4, R e_{p}=9500\right)$.

Fig. 18. Comparison between predicted and measured $S t_{0}$ for different gap ratios $(\delta / D=1.4$, $\left.R e_{p}=9500\right)$.

Fig. 19. Strouhal number in terms of free-stream velocity versus gap ratio for different boundary layers.

Fig. 20. Strouhal number in terms of flow velocity at the pipe center versus gap ratio for different boundary layers. 


\section{Table captions}

Table 1. Characteristics of the different mesh systems.

Table 2. Root-mean-square-error of the simulated mean horizontal velocity profile at different cross-sections.

Table 3. Root-mean-square-error of the simulated mean drag coefficient, mean lift coefficient, RMS lift coefficient and Strouhal number. 


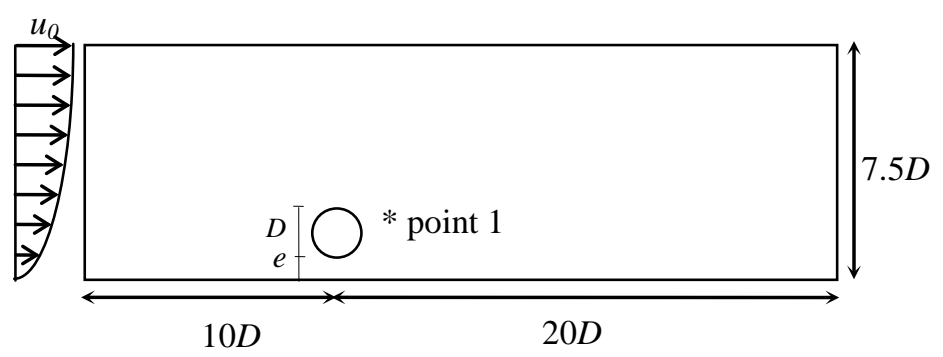

Fig. 1. Sketch of the computational domain. 


\begin{tabular}{|c|c|c|c|}
\hline $4.0 \mathrm{D}$ & $0.3 \times 0.17$ & $0.04 \times 0.17$ & $0.3 \times 0.17$ \\
\hline \multirow[t]{2}{*}{$3.5 \mathrm{D}$} & $0.3 \times 0.03$ & $\begin{array}{l}\text { Subdomain } 1 \\
\bigcirc \quad 0.04 \times 0.03\end{array}$ & $0.3 \times 0.03$ \\
\hline & $6 D$ & $12 D$ & $12 D$ \\
\hline
\end{tabular}

Fig. 2. Computational domain size and dimensionless mesh size in different sections. 


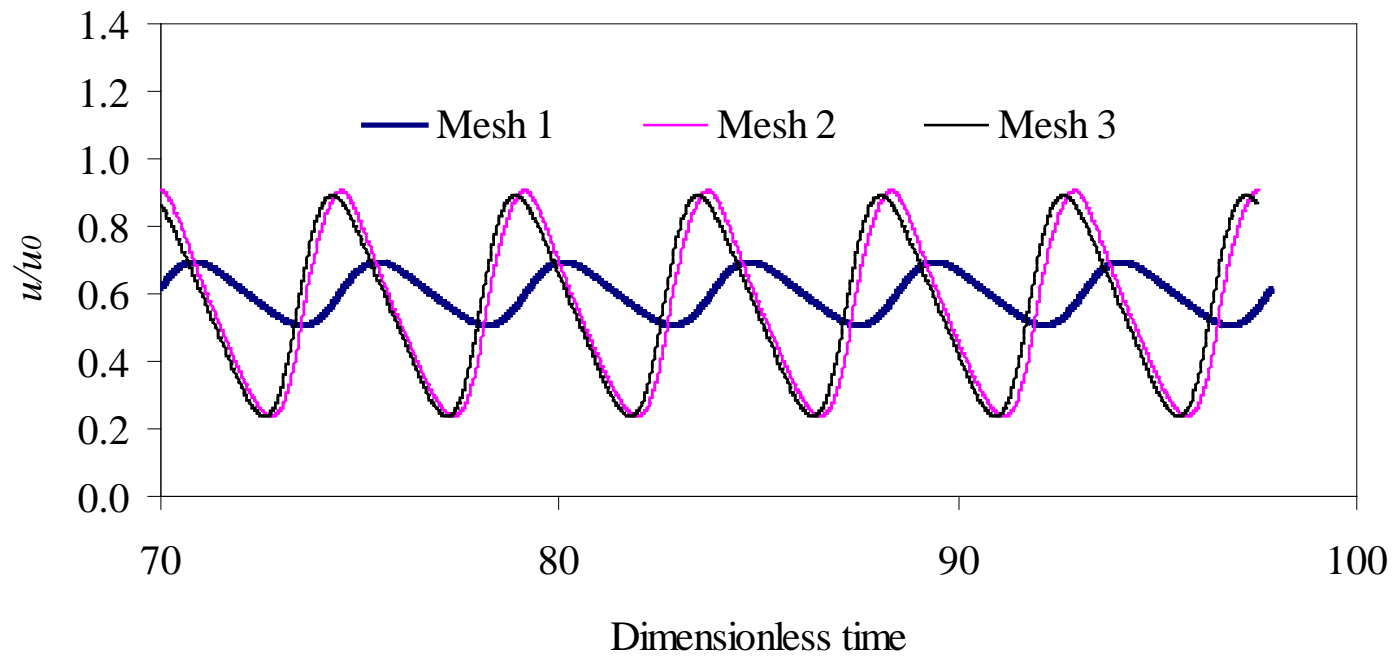

Fig. 3. Simulated instantaneous non-dimensional horizontal velocity at wake flow (point 1) using different mesh resolutions $\left(R e_{p}=9500, e / D=0.5\right)$. 


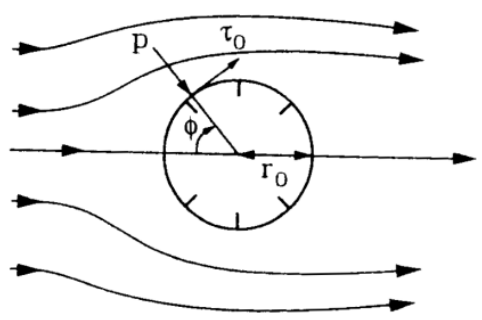

Fig. 4. Total pressure and friction force acting on the pipe [2]. 


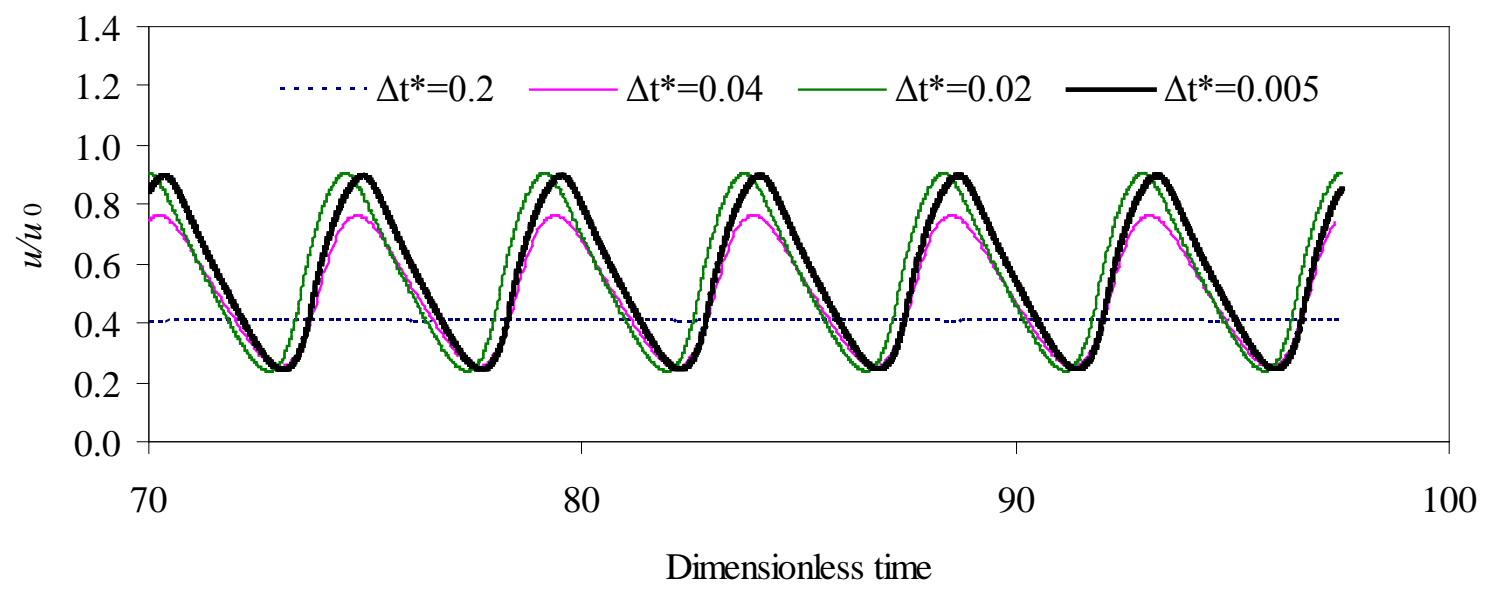

Fig. 5. Simulated instantaneous non-dimensional horizontal velocity at wake flow (point 1) using different time steps in the numerical model $\left(R e_{p}=9500, e / D=0.5\right)$. 


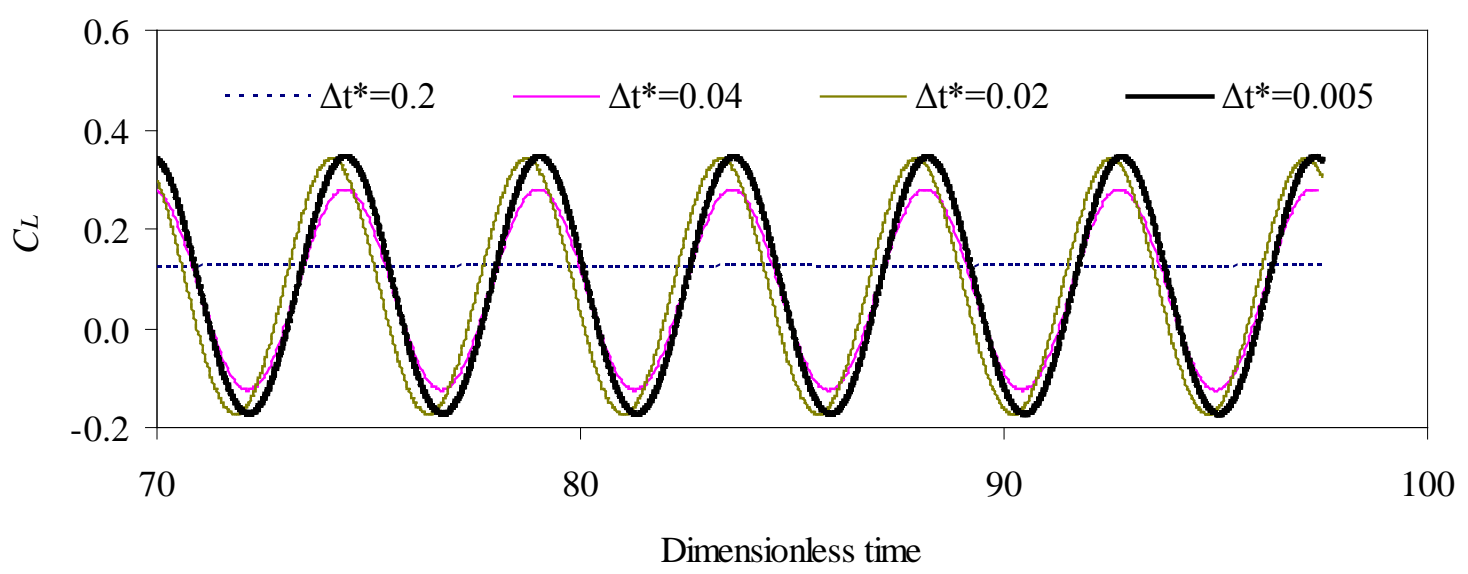

Fig. 6. Simulated instantaneous lift coefficient using different time steps in the numerical model $\left(R e_{p}=9500, e / D=0.5\right)$. 


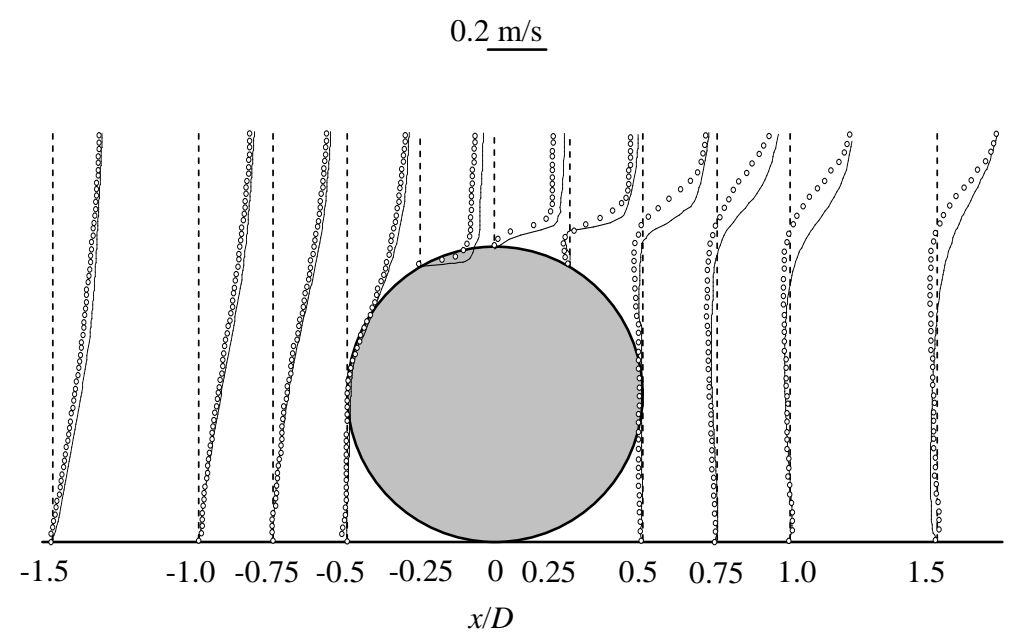

Fig. 7. Mean horizontal velocity. The experimental data of [12] are presented by symbols. The solid lines present the model results ( $\left.e / D=0.0, R e_{p}=9500\right)$. 


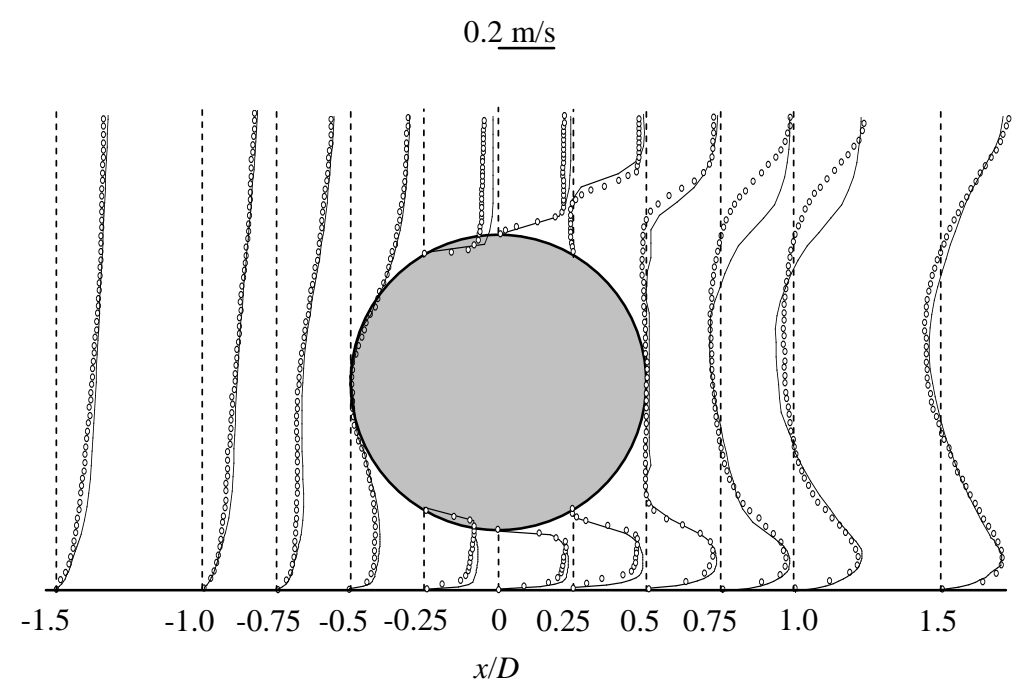

Fig. 8. Mean horizontal velocity. The experimental data of [12] are presented by symbols. The solid lines present the model results $\left(e / D=0.2, R e_{p}=9500\right)$. 

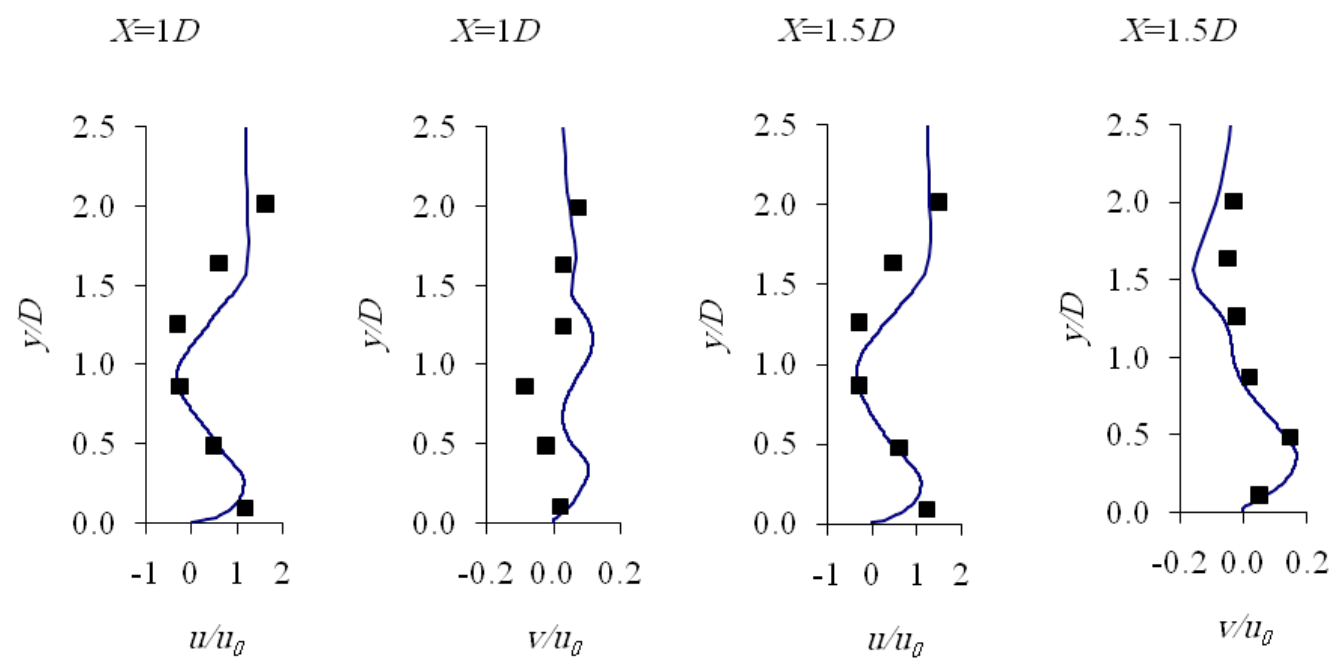

Fig. 9. Mean horizontal and vertical velocity $\left(u / u_{0}\right.$ and $\left.v / u_{0}\right)$. The experimental data of [26] are presented by the square symbols. The solid lines present the model data $(e / D=0.37$, $\left.R e_{p}=7000\right)$. 

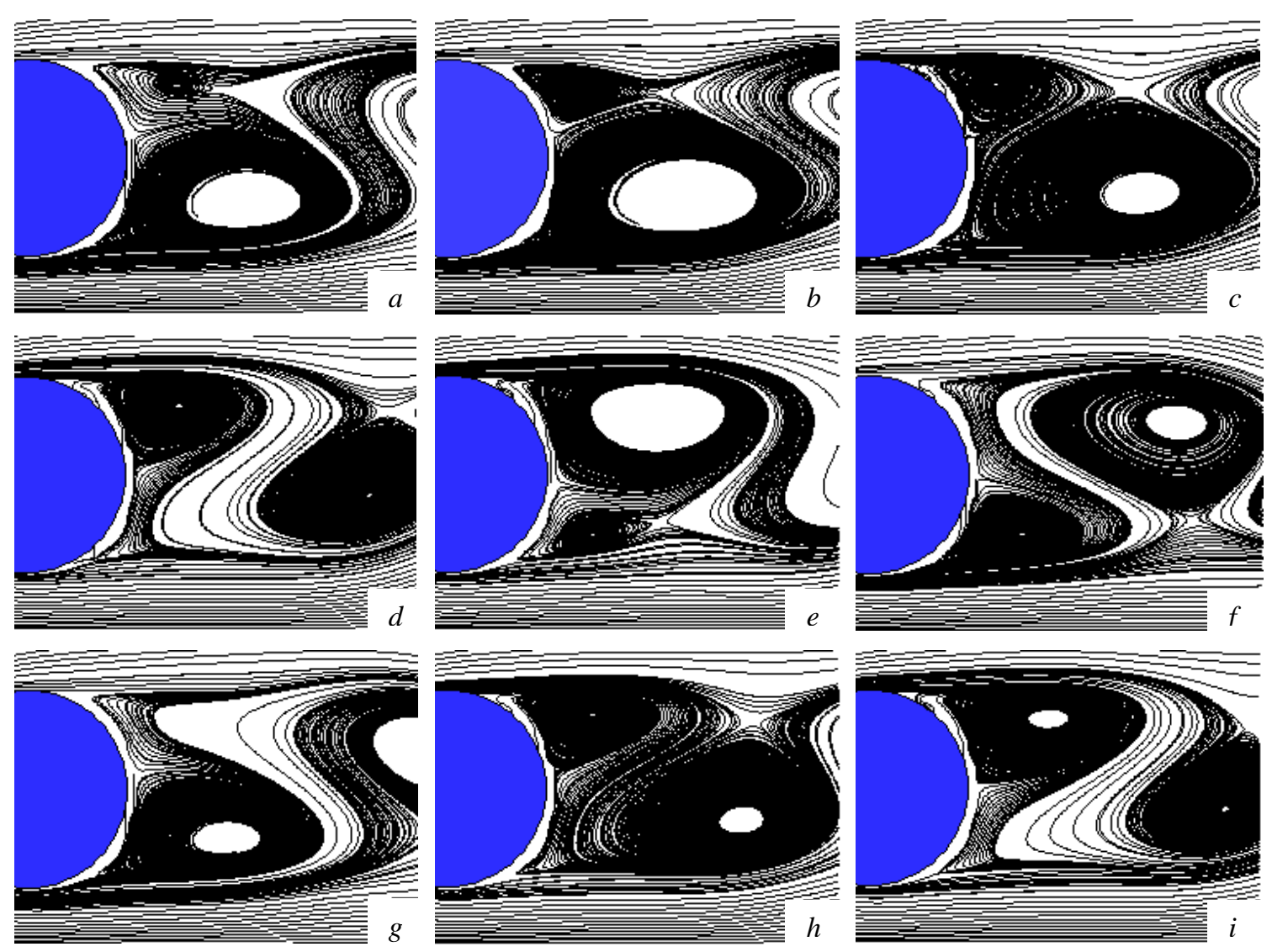

Fig. 10. Instantaneous streamlines and vortex shedding ( $\left.e / D=0.3, R e_{p}=9500\right)$. 


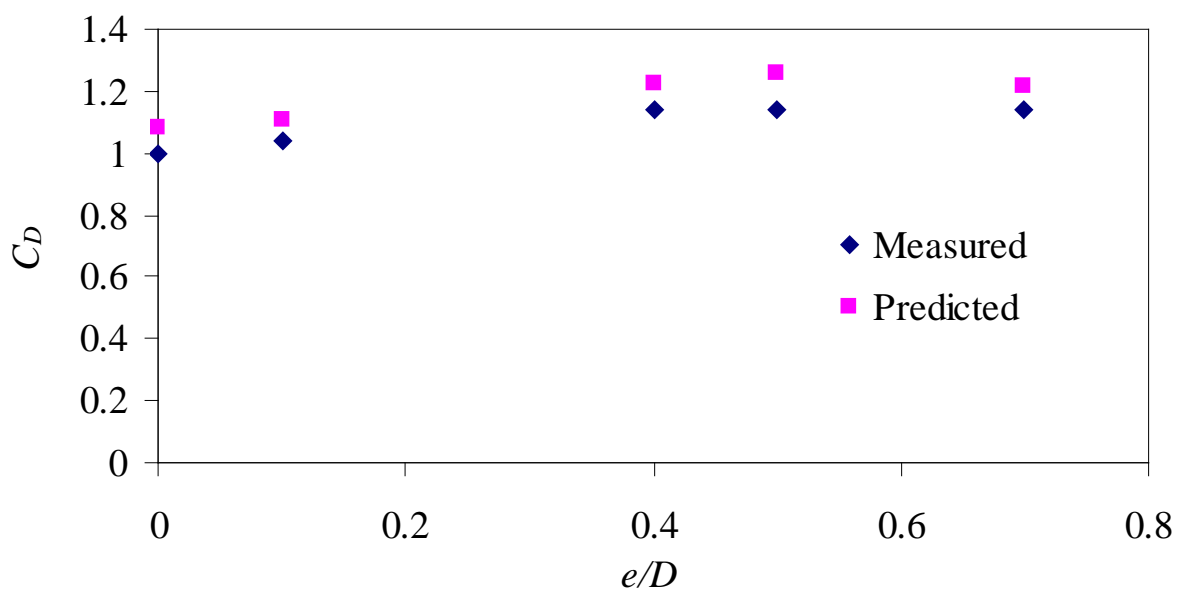

(a)

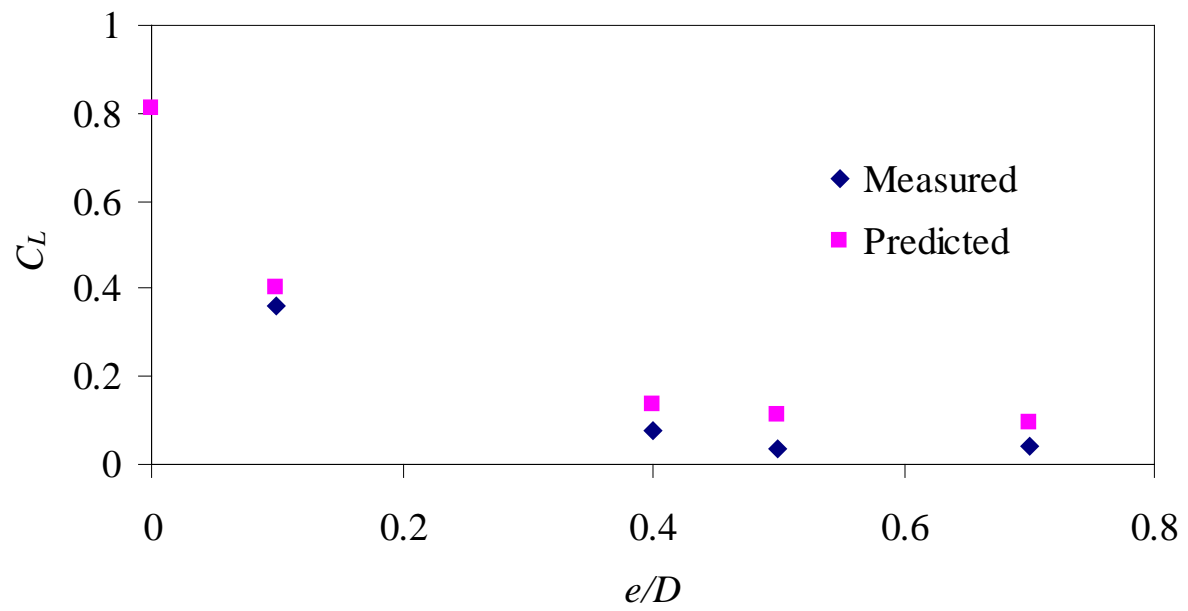

(b) 


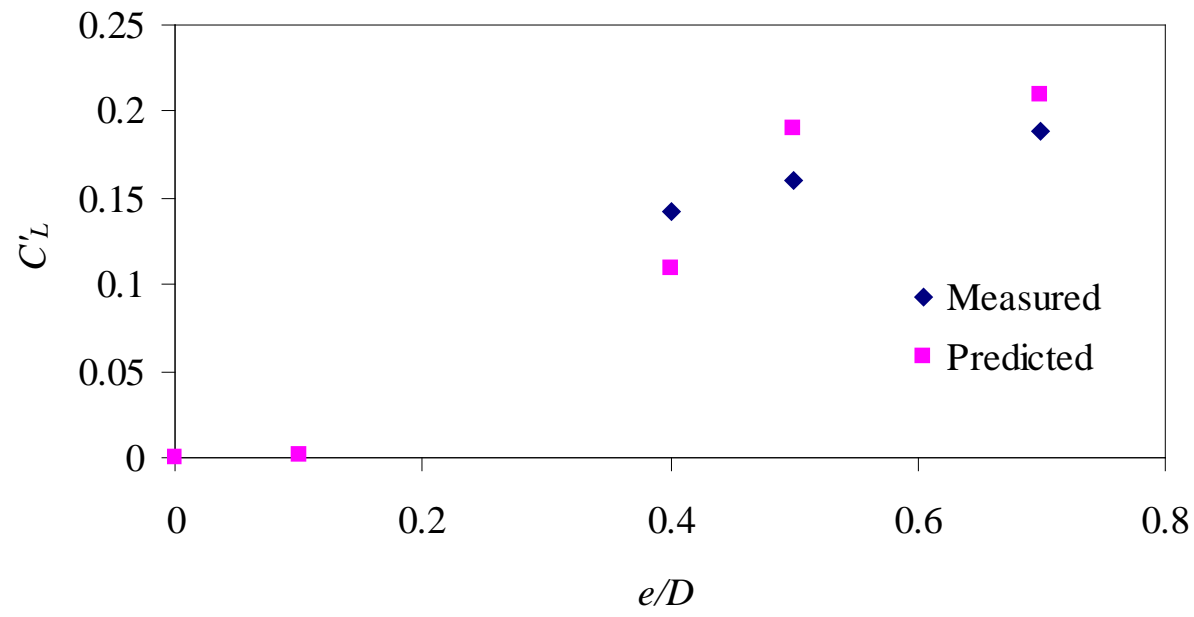

(c)

Fig. 11. Comparison of the (a) mean drag, (b) mean lift and (c) RMS lift coefficients between the present model and measurements of Jensen et al. [9] for different gap ratios. 


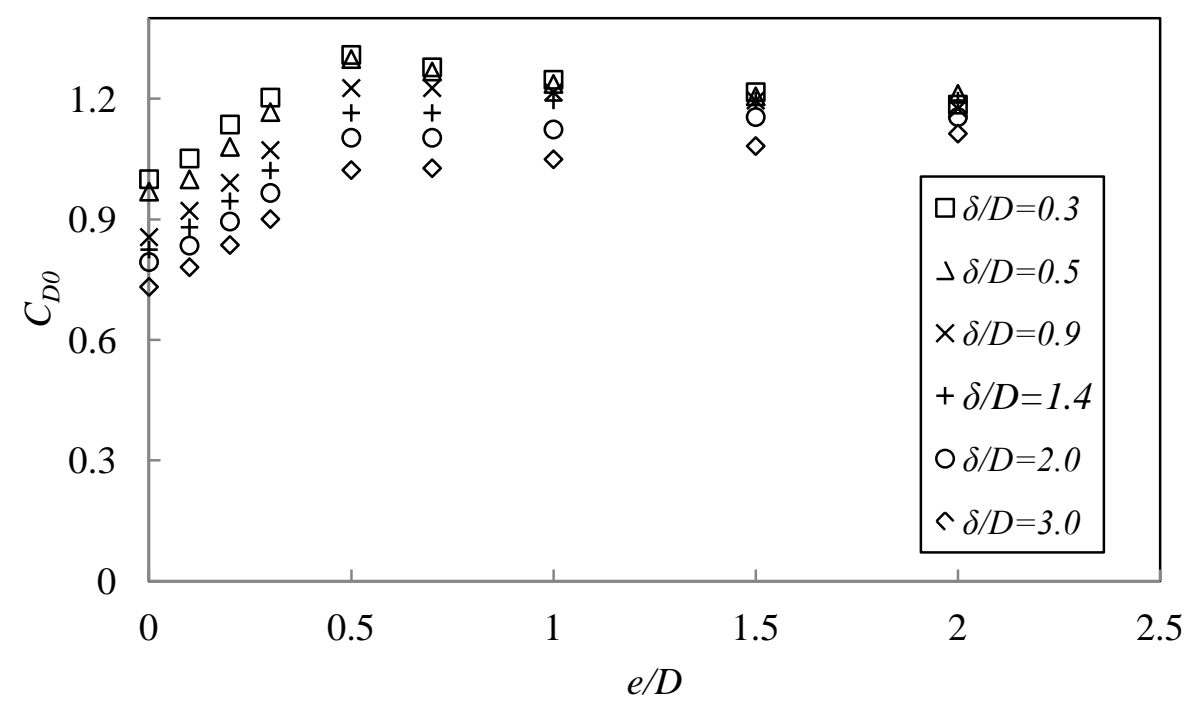

Fig. 12. Drag coefficient in terms of free-stream velocity versus gap ratio for different boundary layers. 


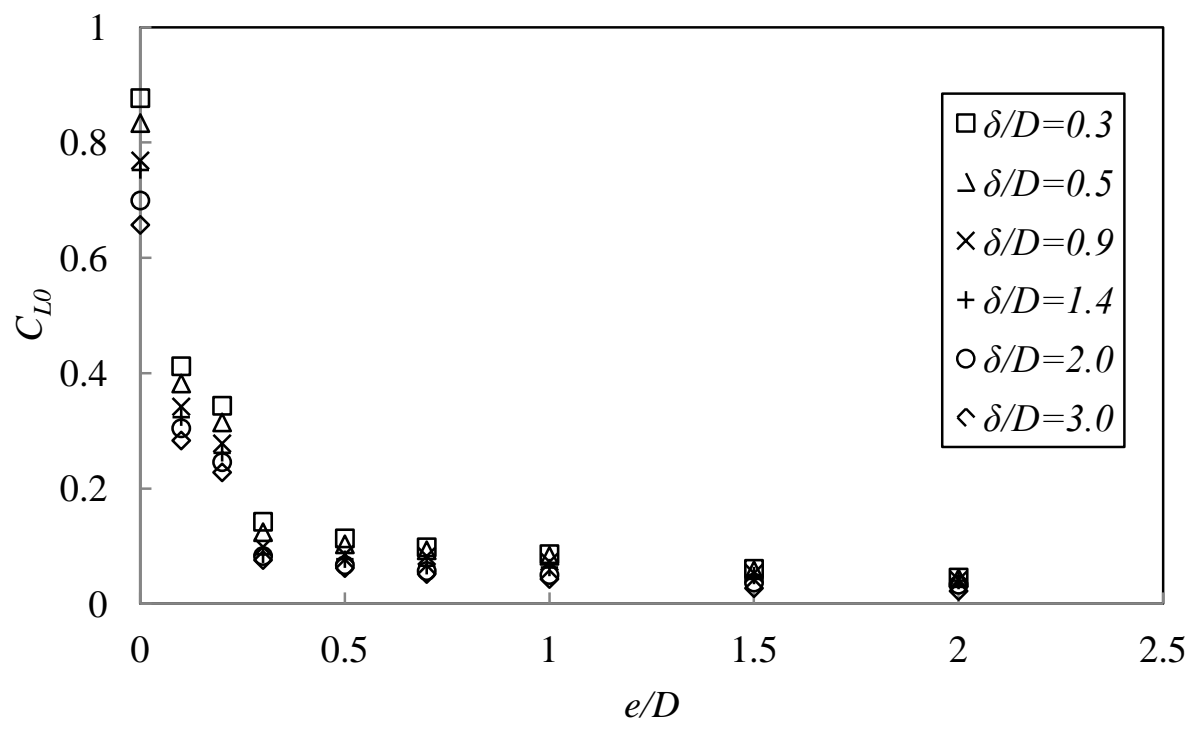

Fig. 13. Lift coefficient in terms of free-stream velocity versus gap ratio for different boundary layers. 


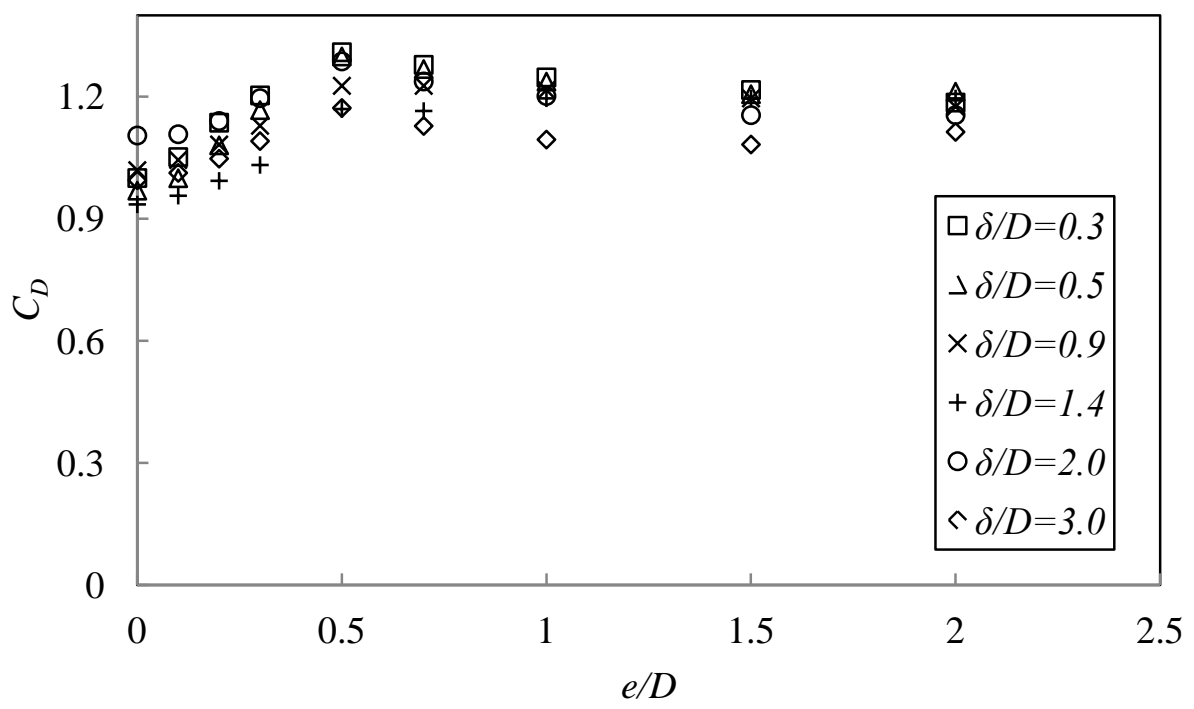

Fig. 14. Drag coefficient in terms of flow velocity at the pipe center versus gap ratio for different boundary layers. 


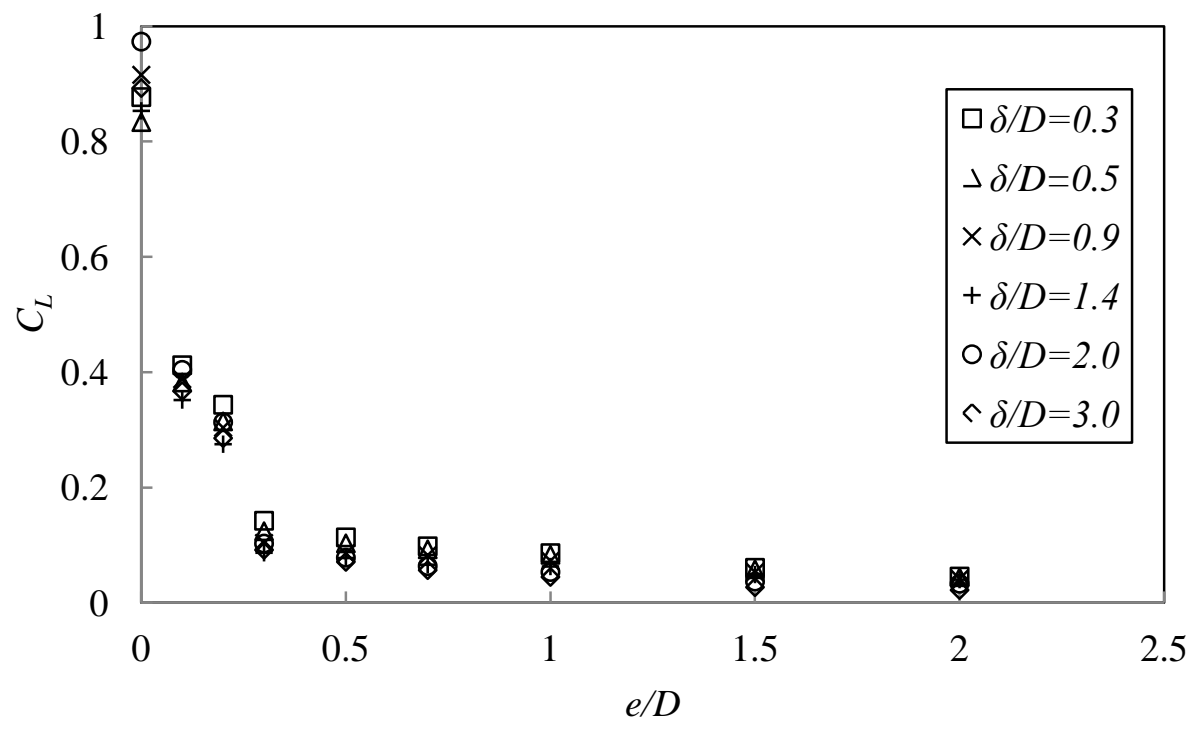

Fig. 15. Lift coefficient in terms of flow velocity at the pipe center versus gap ratio for different boundary layers. 


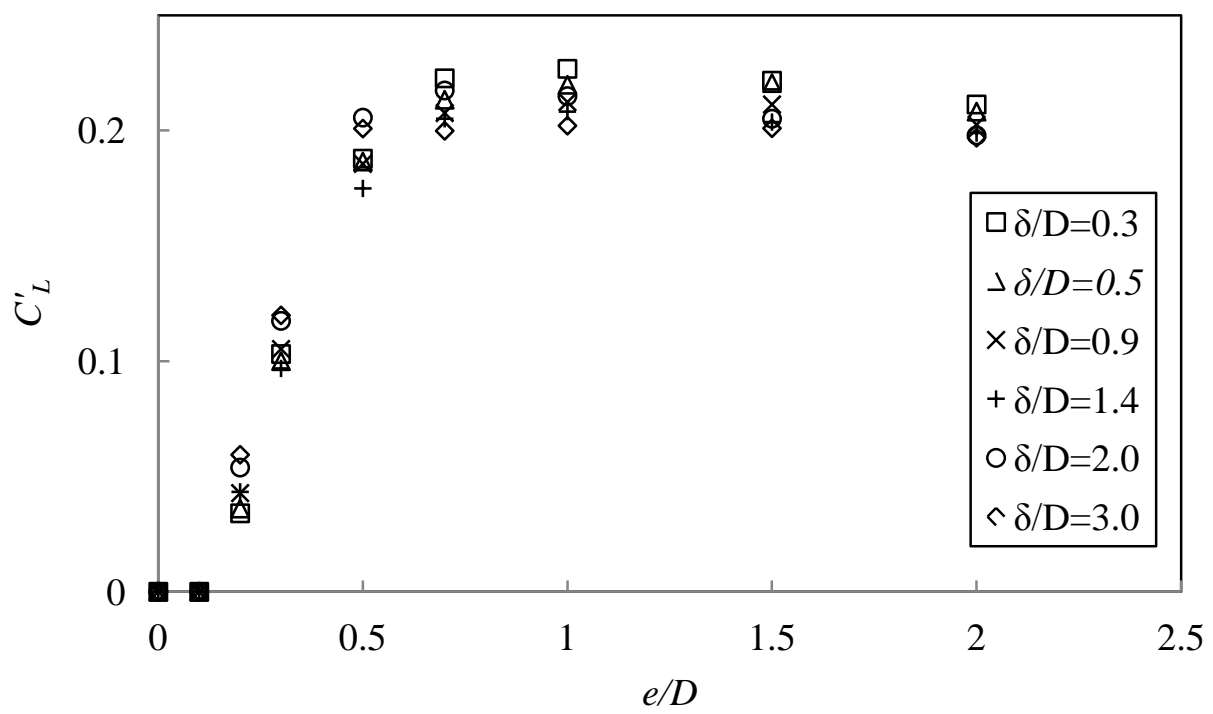

Fig. 16. RMS Lift coefficient in terms of flow velocity at the pipe center versus gap ratio for different boundary layers. 


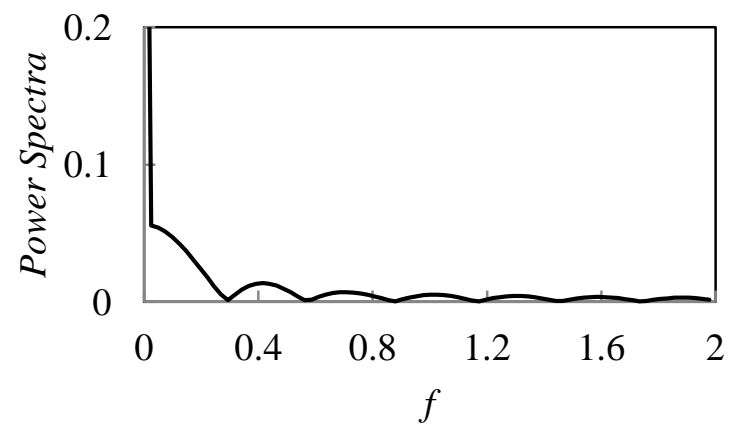

(a)

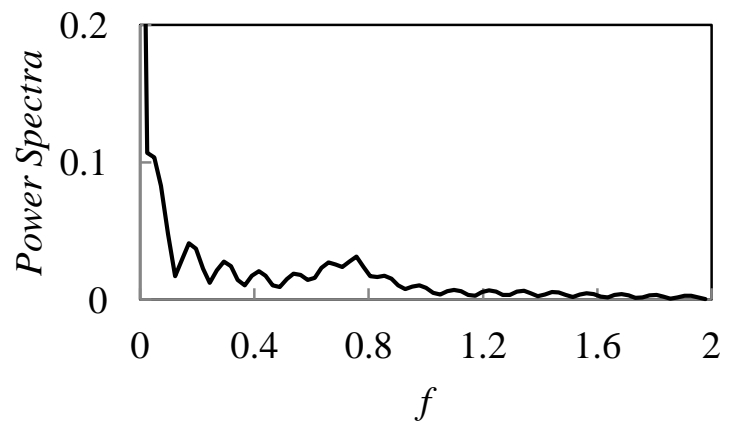

(b)

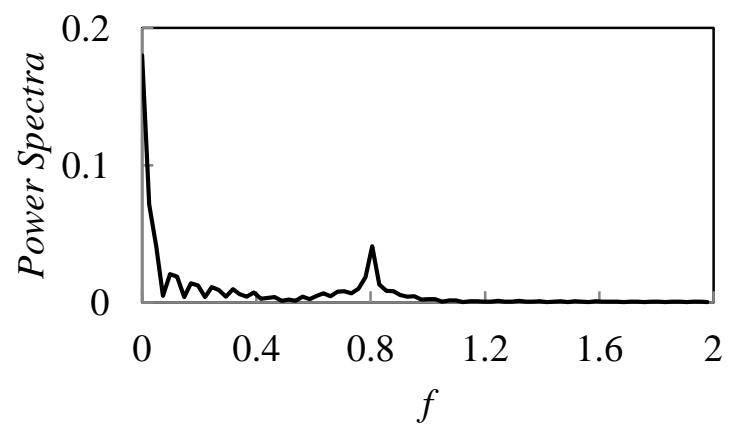

(c) 


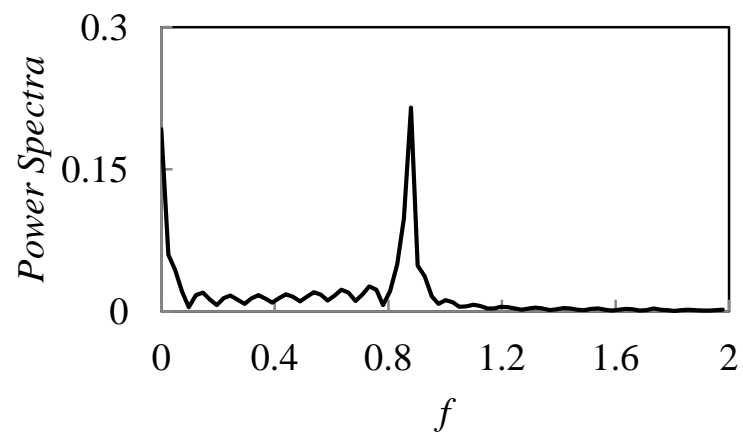

(d)

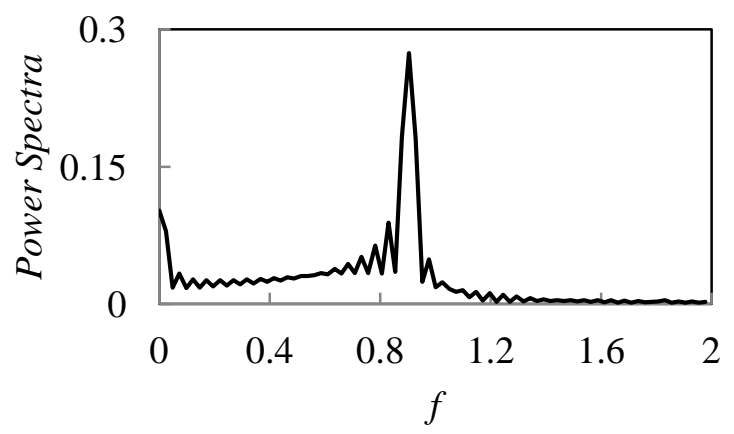

(e)

Fig. 17. Power spectral density of the lift force for $(a) e / D=0.1$, (b) $e / D=0.2$, (c) $e / D=0.3$, (d) $e / D=0.5$ and $(e) e / D=1.0\left(\delta / D=1.4, R e_{p}=9500\right)$. 


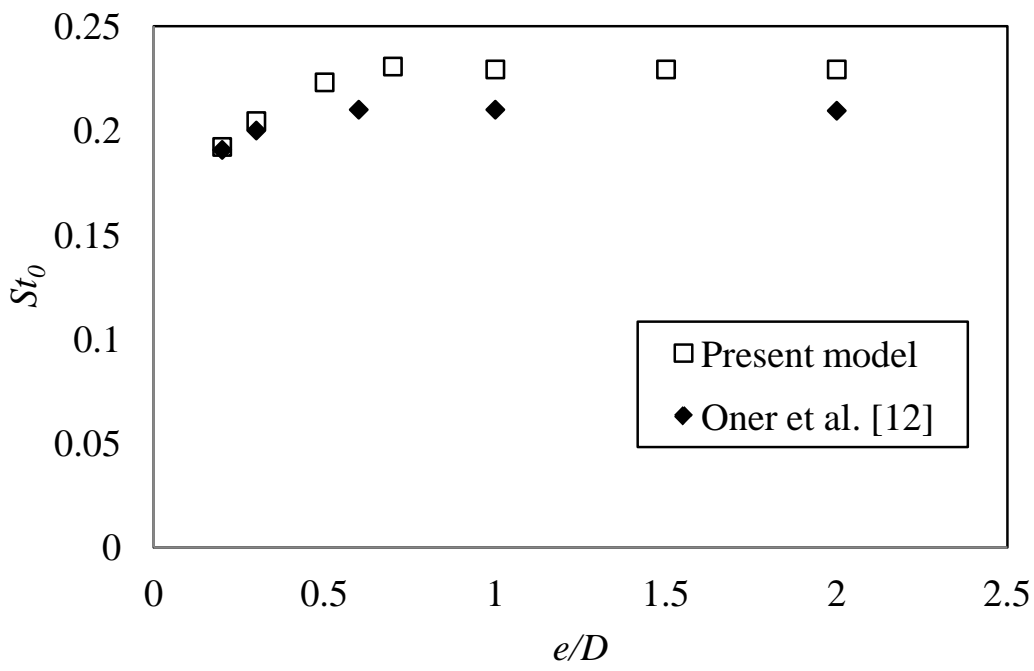

Fig. 18. Comparison between predicted and measured $S t_{0}$ for different gap ratios $(\delta / D=1.4$, $\left.R e_{p}=9500\right)$. 


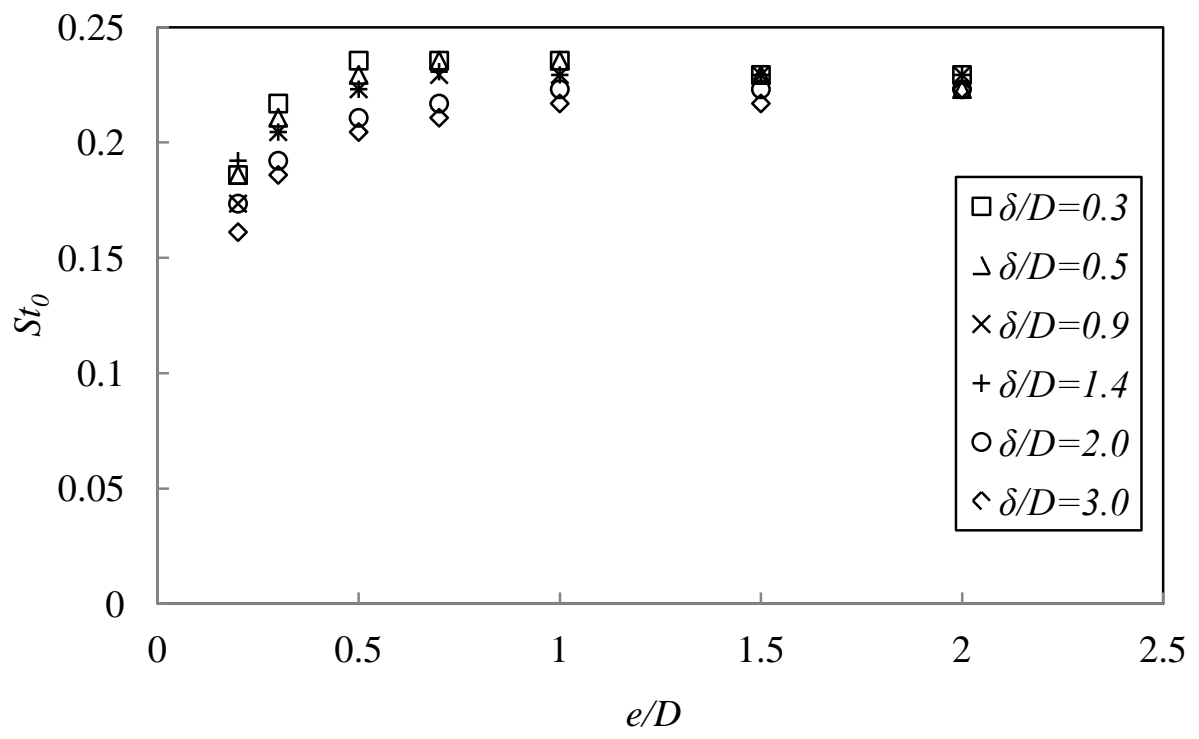

Fig. 19. Strouhal number in terms of free-stream velocity versus gap ratio for different boundary layers. 


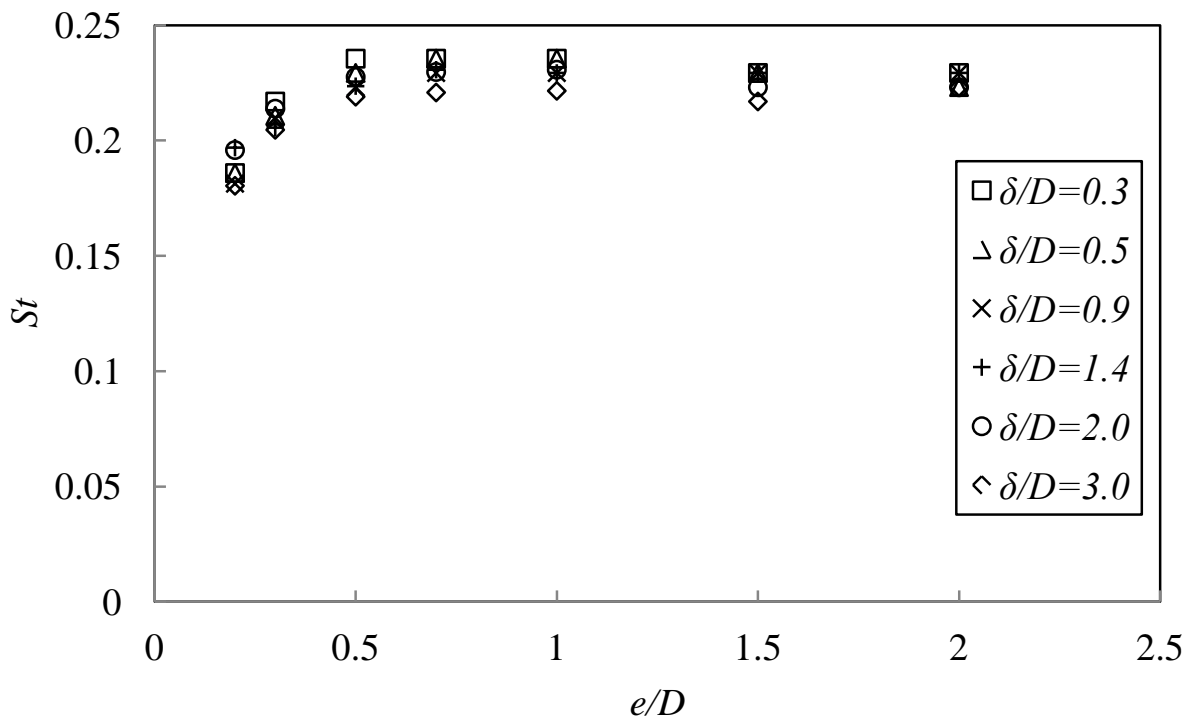

Fig. 20. Strouhal number in terms of flow velocity at the pipe center versus gap ratio for different boundary layers. 
Table 1. Characteristics of the different mesh systems.

\begin{tabular}{cccc}
\hline Mesh & Maximum & Maximum & $\begin{array}{c}\text { Number of mesh per cylinder } \\
\text { name }\end{array}$ \\
& $\boldsymbol{\Delta} \boldsymbol{x}^{*}$ in & $\Delta \boldsymbol{y}^{*}$ in & diameter in vertical and \\
& subdomain $\mathbf{1}$ & subdomain $\mathbf{1}$ & horizontal directions \\
\hline mesh 1 & 0.06 & 0.045 & 25 \\
\hline mesh 2 & 0.04 & 0.03 & 50 \\
\hline mesh 3 & 0.02 & 0.015 & 75 \\
\hline
\end{tabular}


Table 2. Root-mean-square-error of the simulated mean horizontal velocity profile at different cross-sections.

\begin{tabular}{|c|c|c|c|c|c|c|c|c|c|c|c|}
\hline & \multicolumn{11}{|c|}{$\mathrm{RMSE}(\mathrm{cm} / \mathrm{s})$} \\
\hline$x / D$ & -1.5 & -1.0 & -0.75 & -0.5 & -0.25 & $\overline{0}$ & 0.25 & 0.5 & 0.75 & 1.0 & 1.5 \\
\hline$e / D=0$ & 1.78 & 1.3 & 1.28 & 1.18 & 3.0 & 3.3 & 2.24 & 3.35 & 3.59 & 3.64 & 3.19 \\
\hline$e / D=0.2$ & 1.77 & 1.29 & 1.58 & 0.96 & 3.27 & 2.5 & 3.0 & 2.1 & 5.3 & 4.62 & 1.73 \\
\hline
\end{tabular}


Table 3. Root-mean-square-error of the simulated mean drag coefficient, mean lift coefficient, RMS lift coefficient and Strouhal number.

\begin{tabular}{cccc}
\hline Parameter & $\begin{array}{c}\text { Average } \\
\text { measured }\end{array}$ & $\begin{array}{c}\text { Average } \\
\text { predicted }\end{array}$ & RMSE \\
\hline$C_{D}$ & 1.09 & 1.18 & 0.11 \\
\hline$C_{L}$ & 0.13 & 0.24 & 0.13 \\
\hline$C^{\prime}{ }_{L}$ & 0.16 & 0.17 & 0.17 \\
\hline$S_{t_{0}}$ & 0.2 & 0.21 & 0.014 \\
\hline
\end{tabular}

